\title{
VAN EST DIFFERENTIATION AND INTEGRATION
}

\author{
ECKHARD MEINRENKEN AND MARIA AMELIA SALAZAR
}

\begin{abstract}
The classical Van Est theory relates the smooth cohomology of Lie groups with the cohomology of the associated Lie algebra, or its relative versions. Some aspects of this theory generalize to Lie groupoids and their Lie algebroids. In this paper, continuing an idea from 18, we revisit the van Est theory using the Perturbation Lemma from homological algebra. Using this technique, we obtain precise results for the van Est differentiation and integrations maps at the level of cochains. Specifically, we construct homotopy inverses to the van Est differentiation maps that are right inverses at the cochain level.
\end{abstract}

\section{Contents}

1. Introduction

Acknowledgments

2. The Perturbation Lemma

3. Van Est theory for Lie groups

3.1. The van Est double complex

3.2. Differentiation

3.3. Integration

4. Van Est theory for Lie groupoids

4.1. The simplicial manifold $B_{p} G$

4.2. The van Est double complex

4.3. Differentiation

4.4. Integration

4.5. Example: the pair groupoid

5. Van Est maps for Lie groupoid actions on manifolds

5.1. Haar distributions

5.2. The van Est double complex

5.3. Differentiation

5.4. Integration

Appendix A. Proof of Theorem 4.4

References

\begin{tabular}{|l|}
\hline 1 \\
\hline 3 \\
\hline 3 \\
\hline 6 \\
\hline 6 \\
\hline$\frac{8}{9}$ \\
\hline \\
\hline 12 \\
\hline 13 \\
\hline 13 \\
\hline 15 \\
\hline 17 \\
\hline 20 \\
\hline 21 \\
\hline 21 \\
\hline 22 \\
\hline 23 \\
\hline 24 \\
\hline 25 \\
\hline 27 \\
\hline
\end{tabular}

\section{INTRODUCTION}

In a series of papers [27, 28, 29] in the early 1950s, Willem van Est established several key facts relating the smooth group cohomology of a Lie group $G$ to the cohomology of its associated Lie algebra $\mathfrak{g}$. One of his results describes a cochain map $\mathrm{VE}_{G}$ from the Lie group 
complex to the Chevalley-Eilenberg Lie algebra complex, which induces an isomorphism in cohomology up to a certain degree depending on the connectivity properties of $G$. (Using a localized complex, working with germs near the group unit, it induces an isomorphism in all degrees [16, 25]; see also [17].) Furthermore, van Est proved that the smooth group cohomology of a connected Lie group $G$ is canonically isomorphic to the relative Lie algebra cohomology of $\mathfrak{g}$ with respect to the maximal compact subgroup $K$ on $G$. An explicit cochain map from the relative Lie algebra complex $\mathrm{C}^{\bullet}(\mathfrak{g}, K)=\mathrm{C}^{\bullet}(\mathfrak{g})_{K \text {-basic }}$ to the complex $\mathrm{C}^{\bullet}(G)$ was described later by Dupont [11, Shulman-Tischler [24], and Guichardet [14]; see [12, 15, 16, 25] for applications and generalizations. The van Est map was extended by Weinstein-Xu [30] to Lie groupoids $G \rightrightarrows M$, as a cochain map $\mathrm{VE}_{G}: \mathrm{C}^{\bullet}(G) \rightarrow \mathrm{C}^{\bullet}(A)$ from the smooth groupoid cochain complex to the Chevalley-Eilenberg complex of its Lie algebroid $A=\operatorname{Lie}(G)$. Versions of the van Est theorems for Lie groupoids were obtained by Crainic [7]. More recently, an explicit homotopy inverse

$$
R_{G}: C^{\bullet}(A) \rightarrow \mathrm{C}^{\bullet}(G)_{M}
$$

(where the subscript indicates the localized complex) was found by Cabrera-Marcut-Salazar [6].

In this article, we will revisit the van Est theory using the Perturbation Lemma from homological algebra. For the map $\mathrm{VE}_{G}$, this was initiated by Li-Bland and Meinrenken in [18], but we will show that it carries much further. In short, this approach constructs the cochain maps in the van Est theory systematically, from homotopy operators on various double complexes (as opposed to 'guessing' the right formulas). The properties of these cochain maps are obtained from properties of these homotopy operators. This leads to a number of observations that were missed in earlier literature. All our results apply to cochain groups with coefficients in a given $G$-representation $V$, but for simplicity we will only describe the scalar case in the following summary:

Van Est theory for Lie groups. We begin by revisiting the classical setting that $G$ is a Lie group, and $K$ a compact Lie subgroup. We describe a distinguished horizontal homotopy operator on the van Est double complex, and use it to obtain a canonical van Est differentiation map

$$
\mathrm{VE}_{G / K}: \mathrm{C}^{\bullet}(G) \rightarrow \mathrm{C}^{\bullet}(\mathfrak{g})_{K-\text { basic }},
$$

with values in the relative Lie algebra complex. We will show that this relative van Est map is the composition

$$
\mathrm{VE}_{G / K}=\mathrm{VE}_{G} \circ \mathrm{Av}
$$

where Av: $\mathrm{C}(G) \rightarrow \mathrm{C}(G)$ is given on degree $p$ elements by averaging under a natural $K^{p+1}$ action. If $G$ has finitely many components, and $K$ is a maximal compact subgroup, then the diffeomorphism $G / K \cong \mathfrak{g} / \mathfrak{k}$ determines a vertical homotopy operator on the double complex, and a resulting cochain map ('integration')

$$
R_{G / K}: \mathbf{C}^{\bullet}(\mathfrak{g})_{K-\text { basic }} \rightarrow \mathrm{C}^{\bullet}(G) .
$$

This map is similar to (but not equal to) the map defined in [11, 14, 24]. Our theory shows that

$$
\mathrm{VE}_{G / K} \circ R_{G / K}=\mathrm{id}
$$


at the level of cochains. Equations (11) and (2) provide a strengthening of van Est's original results, which are stated at the level of cohomology. For arbitrary compact subgroups $K$ of $G$ (not necessarly maximal compact ones), we have a similar statement for the localized complex; in particular, this applies to $K=\{e\}$.

Van Est maps for Lie groupoids. For a Lie groupoid $G \rightrightarrows M$, with Lie algebroid $A=\operatorname{Lie}(G)$, it was shown in [18] how recover the van Est differentiation map $\mathrm{VE}_{G}$ of [30], through applications of the Perturbation Lemma to the van Est double complex $\mathrm{D}^{\bullet \bullet} \cdot(G)$ from [7. Given a (germ of a) 'tubular structure' for $G$, we also have a vertical homotopy k on the double complex. We will prove that the resulting van Est integration map $R_{G}: \mathrm{C}(A) \rightarrow \mathrm{C}(G)_{M}$, with values in the localized complex, coincides with the integration map of [6]. The fact that $\mathrm{VE}_{G}, R_{G}$ are cochain maps, and that

$$
\mathrm{VE}_{G} \circ R_{G}=\mathrm{id}
$$

on $\mathrm{C}(A)$, are obtained as immediate consequences of the properties of $\mathrm{h}, \mathrm{k}$ and a general algebraic lemma, avoiding the calculations in [30] and [6].

Van Est maps for Lie groupoid actions. Here we consider groupoid actions of $G \rightrightarrows M$ on manifolds $Q$, with anchor map $\Phi: Q \rightarrow M$ a surjective submersion. The Lie algebroid complex $\mathrm{C}(A)$ is generalized to a foliated de Rham complex $\Omega_{\mathcal{F}}(Q)^{G}$ of invariant leafwise forms along the fibers of $\Phi$. (For $Q=G$ with the left action, one recovers $\mathrm{C}(A)$.) According to Crainic [7, if the action is proper, then the choice of a suitable 'Haar distribution' on the action groupoid gives a horizontal homotopy operator $\mathrm{h}$ on a double complex $\mathrm{D}^{\bullet}, \bullet(Q)$. In turn, using the Perturbation Lemma, this determines a differentiation map $\operatorname{VE}_{Q}$ from $\mathrm{C}(G)$ to $\Omega_{\mathcal{F}}(Q)^{G}$. On the other hand, given a right inverse $M \hookrightarrow Q$ to $\Phi$ and a tubular neighborhood embedding, one obtains a vertical homotopy $\mathrm{k}$ and hence an integration map $R_{Q}$ in the opposite direction, at least after localizing. We characterize situations where this integration map is right inverse to differentiation.

Each of the three themes outlined above constitutes a section of this article; these sections are preceded by a quick review of the Perturbation Lemma, which will be our main tool throughout the paper.

Acknowledgments. E.M. was supported by an NSERC Discovery Grant. This study was financed in part by the Coordenação de Aperfeiçoamento de Pessoal de Nível Superior - Brasil (CAPES) - Finance code 001. The authors would like to thank the hospitality of Fields Institute where some of this research was carried out.

\section{The Perturbation Lemma}

Let $\left(\mathrm{D}^{\bullet \bullet \bullet}, \mathrm{d}, \delta\right)$ be a double complex, concentrated in non-negative degrees, 


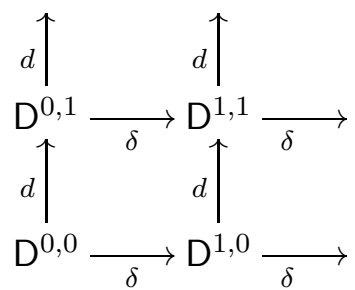

Let $\left(X^{\bullet}, d\right)$ be a cochain complex. A morphism of double complexes

$$
\mathrm{i}: \mathrm{X} \rightarrow \mathrm{D}
$$

(where $\mathbf{X}^{\bullet}$ is regarded as a double complex concentrated in bidegrees $(0, \bullet)$ ) will be called a horizontal augmentation map. Passing to total complexes, i becomes a cochain map from $X^{\bullet}$ to the cochain complex ( $\operatorname{Tot}^{\bullet}(\mathrm{D}), \mathrm{d}+\delta$ ). The Perturbation Lemma, due to Brown [4] and Gugenheim [13, allows us to turn a homotopy operator for the horizontal differential $\delta$ into a homotopy operator with respect to the total differential $\mathrm{d}+\delta$.

Lemma 2.1 (Perturbation Lemma). Suppose $\mathrm{h}: \mathrm{D} \rightarrow \mathrm{D}$ is a linear map of bidegree $(-1,0)$, such that

$$
[\mathrm{h}, \delta]=1-\mathrm{i} \circ \mathrm{p}
$$

for some degree 0 map $\mathrm{p}: \mathrm{D}^{0, \bullet} \rightarrow \mathrm{X}^{\bullet}$. Put $\mathrm{h}^{\prime}=\mathrm{h}(1+\mathrm{dh})^{-1}$ and $\mathrm{p}^{\prime}=\mathrm{p}(1+\mathrm{dh})^{-1}$. Then

$$
\left[\mathrm{h}^{\prime}, \mathrm{d}+\delta\right]=1-\mathrm{i} \circ \mathrm{p}^{\prime} \text {. }
$$

Here, $[\cdot, \cdot]$ denotes the graded commutator, e.g. $[\mathrm{h}, \delta]=\mathrm{h} \delta+\delta \mathrm{h}$.

Proof. Using $\mathrm{h}^{\prime}(1+\mathrm{dh})=\mathrm{h}=(1+\mathrm{hd}) \mathrm{h}^{\prime}$ one finds, by straightforward calculation,

$$
(1+\mathrm{hd})\left[\mathrm{h}^{\prime}, \mathrm{d}+\delta\right](1+\mathrm{dh})=[\mathrm{h}, \mathrm{d}+\delta]=[\mathrm{h}, \mathrm{d}]+1-\mathrm{i} \circ \mathrm{p} .
$$

Expanding $(1+h d)\left(1-\mathrm{i} \circ \mathrm{p}^{\prime}\right)(1+\mathrm{dh})$, using hdi $=\mathrm{hid}=0$, gives the same result.

We shall assume from now on that $\mathrm{p} \circ \mathrm{i}=\mathrm{idx}$, so that $\mathrm{i}$ is injective and $\mathrm{i} \circ \mathrm{p}$ is a projection onto the image of $\mathrm{i}$. Then also $\mathrm{p}^{\prime} \circ \mathrm{i}=\mathrm{idx}$, and $\mathrm{i} \circ \mathrm{p}^{\prime}$ is again a projection. In other words, $\mathrm{i}: \mathrm{X}^{\bullet} \rightarrow \operatorname{Tot}^{\bullet}(\mathrm{D})$ is a homotopy equivalence, with $\mathrm{p}^{\prime}$ a homotopy inverse.

In our applications, there is another cochain complex $\left(\mathrm{Y}^{\bullet}, \delta\right)$, with a vertical augmentation map

$$
\mathrm{j}: \mathrm{Y} \rightarrow \mathrm{D}
$$

(thus $\mathrm{Y}^{\bullet}$ is regarded as a double complex concentrated in bidegrees $(\bullet, 0)$ ). The horizontal homotopy $\mathrm{h}$ allows us to 'invert' the second cochain map in

$$
\mathrm{Y}^{\bullet} \stackrel{\mathrm{j}}{\longrightarrow} \operatorname{Tot}^{\bullet}(\mathrm{D}) \stackrel{\mathrm{i}}{\longleftarrow} \mathrm{X}^{\bullet},
$$

thereby producing a cochain map $\mathrm{p}^{\prime} \circ \mathrm{j}=\mathrm{p} \circ(1+\mathrm{dh})^{-1} \circ \mathrm{j}: \mathrm{Y}^{\bullet} \rightarrow \mathrm{X}^{\bullet}$. On elements of degree $p$, this is given by a 'zig-zag'

$$
(-1)^{p} \mathrm{p} \circ(\mathrm{dh})^{p} \circ \mathrm{j}: \mathrm{Y}^{p} \rightarrow \mathrm{X}^{p}
$$


illustrated here for $p=2$ :

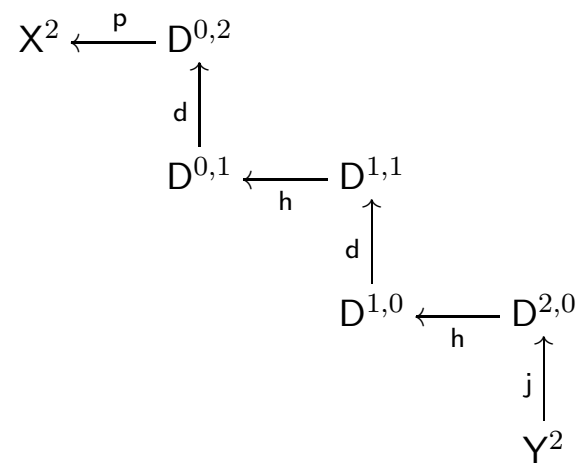

Example 2.2. Let $M$ be a manifold with a covering $\mathcal{U}=\left\{U_{i}\right\}$ by open sets, and let $\mathrm{D}^{p, q}=$ $\mathrm{C}^{p}\left(\mathcal{U}, \Omega^{q}\right)$ be the Cech-de Rham double complex. It comes with a horizontal augmentation map $\mathrm{i}: \Omega^{q}(M) \rightarrow \mathrm{D}^{0, q}$ from the de Rham complex, and a vertical augmentation map j: $\mathrm{C}^{p}(\mathcal{U}, \mathbb{R}) \rightarrow$ $\mathrm{D}^{p, 0}$ from the Cech complex. Given a locally finite partition of unity $\left\{\chi_{i}\right\}$ subordinate to the cover, one obtains a horizontal homotopy operator $\mathrm{h}$, with $\mathrm{p}$ the map taking a collection of $q$-forms $\omega_{i} \in \Omega^{q}\left(U_{i}\right)$ on the open sets to a global $q$-form $\sum_{i} \chi_{i} \omega_{i} \in \Omega^{q}(M)$. See Bott-Tu [3, Proposition 8.5]. The resulting zig-zag (3) defines a Čch-de Rham cochain map $C^{p}(\mathcal{U}, \underline{\mathbb{R}}) \rightarrow$ $\Omega^{p}(M)$, which is nothing but the 'collating formula' of Bott-Tu, [3, Proposition 9.5].

Consider now the situation that the vertical differential has a homotopy operator

$$
\mathrm{k}: \mathrm{D}^{p, q} \rightarrow \mathrm{D}^{p, q-1}, \quad[\mathrm{~d}, \mathrm{k}]=1-\mathrm{j} \circ \mathrm{q},
$$

where $\mathrm{q}: \mathrm{D}^{0, \bullet} \rightarrow \mathrm{Y}^{\bullet}$ is a cochain map for $\mathrm{d}$ with $\mathrm{q} \circ \mathrm{j}=\mathrm{id} \mathrm{Y}$. Then we can apply the Perturbation Lemma 2.1 to this vertical homotopy, and we obtain a cochain map q $\circ(1+\delta \mathrm{k})^{-1} \circ \mathrm{i}: \mathrm{X}^{\bullet} \rightarrow \mathrm{Y}^{\bullet}$ given on degree $p$ elements by a zig-zag,

$$
(-1)^{p} \mathbf{q} \circ(\delta \mathrm{k})^{p} \circ \mathrm{i}: \mathrm{X}^{p} \rightarrow \mathrm{Y}^{p} .
$$

Note that the route taken by the zig-zag (4) retraces the steps of the zig-zag (3). The following result will be used to relate van Est 'integration' and 'differentiation' maps.

Lemma 2.3 (Zig-zag back-and-forth). Suppose the homotopy operators h, k satisfy

$$
\mathrm{h} \circ \mathrm{k}=0, \quad \mathrm{p} \circ \mathrm{k}=0 .
$$

Then (4) followed by (3) is the identity map of $\mathrm{X}^{p}$.

Proof. We first note that

$$
\left.\mathrm{p} \circ \mathrm{j} \circ \mathrm{q}\right|_{\mathrm{D}^{0,0}}=\left.\mathrm{p}\right|_{\mathrm{D}^{0,0}},
$$

and for $p>0$,

$$
\left.\mathrm{h} \circ \mathrm{j} \circ \mathrm{q}\right|_{\mathrm{D}^{p, 0}}=\left.\mathrm{h}\right|_{\mathrm{D}^{p, 0}} .
$$

Equation (7) follows from the calculation, for $p>0$,

$$
\left.\mathrm{h} \circ(1-\mathrm{j} \circ \mathrm{q})\right|_{\mathrm{D}^{p, 0}}=\left.\mathrm{h} \circ[\mathrm{d}, \mathrm{k}]\right|_{\mathrm{D}^{p, 0}}=\left.\mathrm{h} \circ \mathrm{d} \circ \mathrm{k}\right|_{\mathrm{D}^{p, 0}}=0
$$


where we used that $\mathrm{k}$ vanishes on $\mathrm{D}^{p, 0}$ for degree reasons. Equation (6) is obtained similarly. The Lemma now follows for $p=0$ from

$$
\left.\mathrm{p} \circ \mathrm{j} \circ \mathrm{q} \circ \mathrm{i}\right|_{\mathrm{X}^{0}}=\left.\mathrm{p} \circ \mathrm{i}\right|_{\mathrm{X}^{0}}=\mathrm{id} \mathrm{X}^{0},
$$

using (6) , and for $p>0$ from the following calculation, as operators on $\mathrm{X}^{p}$ :

$$
\begin{aligned}
\mathrm{p} \circ(\mathrm{dh})^{p} \circ \mathrm{j} \circ \mathrm{q} \circ(\delta \mathrm{k})^{p} \circ \mathrm{i} & =\mathrm{p} \circ(\mathrm{dh})^{p} \circ(\delta \mathrm{k})^{p} \circ \mathrm{i} \\
& =\mathrm{p} \circ(\mathrm{dh})^{p-1} \circ(1-\mathrm{kd}) \circ(\delta \mathrm{k})^{p-1} \circ \mathrm{i} \\
& =\mathrm{p} \circ(\mathrm{dh})^{(p-1)}(\delta \mathrm{k})^{p-1} \circ \mathrm{i} \\
& =\ldots \\
& =\mathrm{p} \circ \mathrm{i} \\
& =\mathrm{id}_{\mathbf{X}^{p}} .
\end{aligned}
$$

In the first equality, we used (77) if $p>0$, or (6) if $p=0$, to omit the $\mathrm{j} \circ \mathrm{q}$ factor. The second equality follows from

$$
\left.\mathrm{dh} \delta \mathrm{k}\right|_{\mathrm{D}^{p, 0}}=\left.\mathrm{d}(1-\mathrm{ip}-\delta \mathrm{h}) \mathrm{k}\right|_{\mathrm{D}^{p, 0}}=\left.\mathrm{dk}\right|_{\mathrm{D}^{p, 0}}=\mathrm{id}_{\mathrm{D}^{p, 0}}-\left.\mathrm{kd}\right|_{\mathrm{D}^{p, 0}} .
$$

Here we used $\mathrm{hk}=0$ and $\mathrm{pk}=0$.

Remark 2.4. In the Čech-de Rham example 2.2, suppose that the cover $\mathcal{U}$ is a good cover, so that all non-empty intersections of the $U_{i}$ are contractible. Then the choice of such retractions defines a vertical homotopy operator $\mathrm{k}$, and hence gives a cochain map $\Omega^{\bullet}(M) \rightarrow \mathrm{C} \bullet(\mathcal{U}, \mathbb{R})$ in the opposite direction. Unfortunately, the conditions of Lemma 2.3 are not satisfied, in general; hence this map won't give a right inverse to the Čech-de Rham cochain map, even though it is a homotopy inverse.

\section{VAN EST THEORY FOR LIE GROUPS}

Suppose that $G$ is a Lie group with finitely many connected components. One of van Est's results, often referred to as the van Est theorem, is that the smooth cohomology of $G$ with coefficients in a representation $V$ is canonically isomorphic to the relative Lie algebra cohomology with respect to a maximal compact subgroup $K \subseteq G$, with coefficients in $V$. As we will see, the Perturbation Lemma will guide us towards explicit van Est maps, in both directions. Furthermore, we will show that the 'integration map' is a right inverse to the 'differentiation map', at the level of cochains.

3.1. The van Est double complex. Let $G$ be a Lie group, with a representation on a vector space $V$. The smooth Lie group cochain complex $\left(\mathrm{C}^{\bullet}(G, V), \delta\right)$ has as its $p$-cochains the functions

$$
C^{p}(G, V)=C^{\infty}\left(G^{p}, V\right)
$$

and the differential is given by

$$
\begin{aligned}
(\delta f)\left(g_{1}, \ldots, g_{p+1}\right)= & f\left(g_{2}, \ldots, g_{p+1}\right)+\sum_{i=1}^{p}(-1)^{i} f\left(g_{1}, \ldots, g_{i} g_{i+1}, \ldots, g_{p+1}\right) \\
& +(-1)^{p+1}\left(g_{p+1}\right)^{-1} \cdot f\left(g_{1}, \ldots, g_{p}\right) .
\end{aligned}
$$


On the other hand, letting $\mathfrak{g}=\operatorname{Lie}(G)$, we have the usual Lie algebra complex $\left(\mathrm{C}^{\bullet}(\mathfrak{g}, V), \mathrm{d}_{C E}\right)$, where

$$
\mathrm{C}^{q}(\mathfrak{g}, V)=V \otimes \wedge^{q} \mathfrak{g}^{*},
$$

and where $\mathrm{d}_{C E}$ is the Chevalley-Eilenberg differential. The Lie group complex and Lie algebra complex are related by a double complex $\left(\mathrm{D}^{\bullet} \bullet(G, V), \delta, \mathrm{d}\right)$ introduced in van Est's original articles [27, 28, 29]; see also Guichardet [14]. The van Est double complex has bigraded components

$$
\mathrm{D}^{p, q}(G, V)=C^{\infty}\left(G^{p} \times G, V \otimes \wedge^{q} \mathfrak{g}^{*}\right)
$$

and the horizontal differential is given by

$$
\begin{aligned}
(\delta \psi)\left(g_{1}, \ldots, g_{p+1} ; g\right)= & \psi\left(g_{2}, \ldots, g_{p+1} ; g\right)+\sum_{i=1}^{p}(-1)^{i} \psi\left(g_{1}, \ldots, g_{i} g_{i+1}, \ldots, g_{p+1} ; g\right) \\
& +(-1)^{p+1} \psi\left(g_{1}, \ldots, g_{p} ; g_{p+1} g\right) .
\end{aligned}
$$

The vertical differential is given by $\mathrm{d}=(-1)^{p} \mathrm{~d}_{C E}$, where we identify $\mathrm{D}^{p, \bullet}(G, V)$ with the Chevalley-Eilenberg complex of the $\mathfrak{g}$-representation on $C^{\infty}\left(G^{p} \times G\right) \otimes V$, using the infinitesimal $\mathfrak{g}$-representation on $V$ and the representation $\xi \mapsto\left(0, \xi^{L}\right)$ (the left-invariant vector field on the last $G$-factor) on $C^{\infty}\left(G^{p} \times G\right)$. Then $[\mathrm{d}, \delta]=\mathrm{d} \delta+\delta \mathrm{d}=0$, as desired. This double complex has horizontal and vertical augmentation maps

$$
\mathrm{i}: \mathrm{C}^{\bullet}(\mathfrak{g}, V) \rightarrow \mathrm{D}^{0, \bullet}(G, V), \quad \mathrm{j}: \mathrm{C}^{\bullet}(G, V) \rightarrow \mathrm{D}^{\bullet, 0}(G, V),
$$

where $\mathrm{i}$ is the inclusion of constant functions, while

$$
(\mathrm{j} f)\left(g_{1}, \ldots, g_{p} ; g\right)=g^{-1} \cdot f\left(g_{1}, \ldots, g_{p}\right)
$$

for $f \in \mathrm{C}^{p}(G, V)=C^{\infty}\left(G^{p}, V\right)$. Note $\mathrm{j}$ is an inclusion of $\mathrm{C}(G, V)$ as the $G$-invariant part of $\mathrm{D}^{\bullet}, 0(G, V)$, or equivalently the $G$-basic part of the full double complex, with respect to the following $G$-action

$$
(a \cdot \psi)\left(g_{1}, \ldots, g_{p} ; g\right)=a \cdot \psi\left(g_{1}, \ldots, g_{p} ; g a\right)
$$

(using the coadjoint action on $\wedge \mathfrak{g}^{*}$ ).

Now suppose $K \subseteq G$ is a compact Lie subgroup. The relative Lie algebra complex with coefficients in $V$ is the $K$-basic subcomplex

$$
\mathrm{C}(\mathfrak{g}, V)_{K \text {-basic }}
$$

That is, it consists of elements that are annilhilated by contractions with elements of $\mathfrak{k}$ on the $\wedge \mathfrak{g}^{*}$-factor, and are invariant for the action of $K \subseteq G$. Similarly, we can consider the $K$-basic sub-double complex $\mathrm{D}(G, V)_{K \text {-basic }}$ with respect to the action (10). The two augmentation maps for $\mathrm{D}(G, V)$ restrict to augmentation maps

$$
\text { i : } \mathrm{C}^{\bullet}(\mathfrak{g}, V)_{K \text {-basic }} \rightarrow \mathrm{D}^{0, \bullet}(G, V)_{K \text {-basic }}, \quad \mathrm{j}: \mathrm{C}^{\bullet}(G, V) \rightarrow \mathrm{D}^{\bullet, 0}(G, V)_{K \text {-basic }}
$$


3.2. Differentiation. The double complex $\mathrm{D}(G, V)_{K \text {-basic }}$ has a horizontal homotopy operator:

$$
(\mathrm{h} \psi)\left(g_{1}, \ldots, g_{p-1} ; g\right)=(-1)^{p} \int_{K} \psi\left(g_{1}, \ldots, g_{p-1}, g k^{-1} ; k\right) \mathrm{d} k
$$

here $\mathrm{d} k$ is the normalized invariant Haar measure on $K$. Indeed, a direct calculation shows that $\delta \mathrm{h}+\mathrm{h} \delta=1-\mathrm{i} \circ \mathrm{p}$ where $\mathrm{p}$ vanishes on elements of bidegree $(p, q)$ with $p>0$, while

$$
\mathrm{p}(\psi)=\int_{K} \psi(k) \mathrm{d} k,
$$

for $\psi \in \mathrm{D}^{0, q}(G, V)_{K \text {-basic }}=C^{\infty}\left(G, V \otimes \wedge^{q} \mathfrak{g}^{*}\right)_{K \text {-basic }}$.

Remark 3.1. For $K=\{e\}$, the homotopy operator simplifies to

$$
(\mathrm{h} \psi)\left(g_{1}, \ldots, g_{p-1} ; g\right)=(-1)^{p} \psi\left(g_{1}, \ldots, g_{p-1}, g ; e\right) \text {. }
$$

Note that this homotopy operator on $\mathrm{D}(G, V)$ does not preserve the basic subcomplex with respect to a nontrivial compact subgroup.

Using the Perturbation Lemma 2.1, we obtain a cochain map (van Est differentiation)

$$
\mathrm{VE}_{G / K}=\mathrm{p} \circ(1+\mathrm{dh})^{-1} \circ \mathrm{j}: \mathrm{C}^{\bullet}(G, V) \rightarrow \mathrm{C}^{\bullet}(\mathfrak{g}, V)_{K \text {-basic }} .
$$

For an explicit description of this map, consider the following $p+1$ commuting $G$-actions on the space $C^{\infty}\left(G^{p}, V\right)$,

$$
(a \cdot f)\left(g_{1}, \ldots, g_{p}\right)= \begin{cases}f\left(a^{-1} g_{1}, \ldots, g_{p}\right) & i=0, \\ f\left(g_{1}, \ldots, g_{i} a, a^{-1} g_{i+1}, \ldots, g_{p}\right) & 0<i<p, \\ a \cdot f\left(g_{1}, \ldots, g_{p} a\right) & i=p .\end{cases}
$$

For each of these actions we can consider its restriction to $K$; denote by

$$
\operatorname{Av}^{(i)}: C^{\infty}\left(G^{p}, V\right) \rightarrow C^{\infty}\left(G^{p}, V\right)
$$

the averaging operation with respect to $i$-th $K$-action. The operators $\mathrm{Av}^{(i)}$ commute since the actions commute, and we denote by $\mathrm{Av}=\mathrm{Av}^{(0)} \circ \cdots \circ \mathrm{Av}^{(p)}$ the total $K^{p+1}$-averaging operation. We will also need the $G$-actions, obtained from the actions (13) labeled $i, i+1, \ldots, p$ by passing to the diagonal action:

$$
(a \cdot f)\left(g_{1}, \ldots, g_{p}\right)=a \cdot f\left(g_{1}, \ldots, g_{i} a, a^{-1} g_{i+1} a, \ldots, a^{-1} g_{p} a\right) .
$$

Denote by $\mathrm{d}^{(i)}$ the corresponding Chevalley-Eilenberg differential on $C^{\infty}\left(G^{p}, V\right) \otimes \wedge \mathfrak{g}^{*}$.

Theorem 3.2. For any compact Lie subgroup $K \subseteq G$, the van Est differentiation $\mathrm{VE}_{G / K}=$ $\mathrm{p} \circ(1+\mathrm{dh})^{-1} \circ \mathrm{j}$ is given by the formula

$$
\mathrm{VE}_{G / K}(f)=\left.\left(\mathrm{d}^{(1)} \cdots \mathrm{d}^{(p)} \operatorname{Av}(f)\right)\right|_{(e, \ldots, e)},
$$

for $f \in C^{\infty}\left(G^{p}, V\right)$. In particular, $\mathrm{VE}_{G / K}=\mathrm{VE}_{G} \circ \mathrm{Av}$. 
Proof. The $G$-actions on $C^{\infty}\left(G^{p}, V\right)$, given by Equation (13), extend to commuting actions on $C^{\infty}\left(G^{p}, V \otimes \wedge^{q} \mathfrak{g}^{*}\right)$, given by the same formulas (replacing $V$ with $\left.V \otimes \wedge^{q} \mathfrak{g}^{*}\right)$. Denote by $\mathrm{Av}^{(i)}$ the averaging operation on $C^{\infty}\left(G^{p}, V \otimes \wedge^{q} \mathfrak{g}^{*}\right)$ for the $i$-th action of $K \subseteq G$.

We calculate $\mathrm{VE}_{G / K}=(-1)^{p} \mathrm{p} \circ(\mathrm{dh})^{p} \circ \mathrm{j}$ on $f \in \mathrm{C}^{p}(G, V)$. The first few steps are

$$
\begin{aligned}
(-1)^{p}(\mathrm{j} f)\left(g_{1}, \ldots, g_{p} ; g\right) & =(-1)^{p} g^{-1} \cdot f\left(g_{1}, \ldots, g_{p}\right) \\
(-1)^{p}(\mathrm{hj} f)\left(g_{1}, \ldots, g_{p-1} ; g\right) & =\int_{K} k^{-1} \cdot f\left(g_{1}, \ldots, g_{p-1}, g k^{-1}\right) \mathrm{d} k \\
& =\left(\mathrm{Av}{ }^{(p)} f\right)\left(g_{1}, \ldots, g_{p-1}, g\right) \\
(-1)^{p}(\operatorname{dhj} f)\left(g_{1}, \ldots, g_{p-1} ; g\right) & =(-1)^{p-1}\left(\mathrm{~d}^{(p)} \mathrm{Av}{ }^{(p)} f\right)\left(g_{1}, \ldots, g_{p-1}, g\right) .
\end{aligned}
$$

In the last line, we used that the $G$-action (10) defining $\mathrm{d}_{C E} \psi$ for

$$
\psi\left(g_{1}, \ldots, g_{p-1} ; g\right)=\left(\mathrm{Av}^{(p)} f\right)\left(g_{1}, \ldots, g_{p-1}, g\right)
$$

corresponds to the $p$-th $G$-action (13) (after setting $g_{p}=g$ ), defining d ${ }^{(p)}$. Next,

$$
\begin{aligned}
(-1)^{p}(\operatorname{hdhj} f)\left(g_{1}, \ldots, g_{p-2} ; g\right) & =\int_{K}\left(\mathrm{~d}^{(p)} \mathrm{Av}^{(p)} f\right)\left(g_{1}, \ldots, g_{p-2}, g k^{-1}, k\right) \mathrm{d} k \\
& =\left(\mathrm{Av}^{(p-1)} \mathrm{d}^{(p)} \mathrm{Av}^{(p)} f\right)\left(g_{1}, \ldots, g_{p-2}, g, e\right) \\
(-1)^{p}(\operatorname{dhdhj} f)\left(g_{1}, \ldots, g_{p-2} ; g\right) & =\left(\mathrm{d}^{(p-1)} \mathrm{Av}^{(p-1)} \mathrm{d}^{(p)} \mathrm{Av}^{(p)} f\right)\left(g_{1}, \ldots, g_{p-2}, g, e\right) .
\end{aligned}
$$

Here we used that the $G$-action (10) defining $\mathrm{d}_{C E} \psi$ for

$$
\psi\left(g_{1}, \ldots, g_{p-2} ; g\right)=\left(\mathrm{Av}^{(p-1)} \mathrm{d}^{(p)} \operatorname{Av}^{(p)} f\right)\left(g_{1}, \ldots, g_{p-2}, g, e\right)
$$

corresponds (for $g_{p-1}=g, g_{p}=e$ ) to the $(p-1)$-st diagonal $G$-action (14), defining $\mathrm{d}^{(p-1)}$. Continuing in this fashion, we arrive at

$$
(-1)^{p} \mathrm{p} \circ(\mathrm{dh})^{p} \circ \mathrm{j} f=\left(\operatorname{Av}^{(0)} \mathrm{d}^{(1)} \mathrm{Av}^{(1)} \cdots \mathrm{d}^{(p)} \operatorname{Av}^{(p)} f\right)(e, \cdots, e) .
$$

Since the $i$-th-action (13) commutes with the $j$-th action (14) for $i<j$, the operator $\mathrm{Av}^{(i)}$ commutes with $\mathrm{d}^{(j)}$ for $i<j$. Hence we may move the averaging operations all the way to the right, resulting in the formula (15).

Remark 3.3. While there are various more or less explicit descriptions of the van Est differentiation (see in particular [14]), we are not aware of an appearance of the formula (15) in the literature, for general compact $K$. Note also that it is not necessary to pass to a 'normalized subcomplex'.

3.3. Integration. For the discussion of van Est integration maps, another interpretation of the relative (double) complex will be convenient. Let $G$ act on itself by left translation $g \mapsto a g$, and let $\Omega^{q}(G, V)^{G}$ be the corresponding complex of $G$-invariant $V$-valued forms, where the action of $V$ is the given representation. Restriction of such a form to the group unit gives an isomorphism $\Omega^{q}(G, V)^{G} \rightarrow V \otimes \wedge^{q} \mathfrak{g}^{*}$, which intertwines the de Rham differential and the Chevalley-Eilenberg differential. Thus

$$
\mathrm{C}^{q}(\mathfrak{g}, V) \cong \Omega^{q}(G, V)^{G}
$$

as differential complexes. The $G$-action on $C^{q}(\mathfrak{g}, V)=V \otimes \wedge^{q} \mathfrak{g}^{*}$ (with the given $G$-representation on $V$ and the coadjoint action on $\left.\wedge \mathfrak{g}^{*}\right)$ corresponds to the action on $\Omega^{q}(G, V)^{G}$ coming from 
the action $g \mapsto g a^{-1}$ on $G$ and the trivial action on $V$. Consider the restriction of this action to $K$; Since $\Omega^{q}(G, V)_{K \text {-basic }}=\Omega^{q}(G / K, V)$, and similarly for the invariant forms for the action by left multiplication, we obtain the identification

$$
\mathrm{C}^{q}(\mathfrak{g}, V)_{K \text {-basic }} \cong \Omega^{q}(G / K, V)^{G} .
$$

Similarly, elements $\psi \in \mathrm{D}^{p, q}(G, V)_{K \text {-basic may be identified with functions }}$

$$
\beta: G^{p} \rightarrow \Omega^{q}(G / K, V),
$$

with smooth dependence on $\left(g_{1}, \ldots, g_{p}\right) \in G^{p}$ as parameters. In these terms, the two differentials are

$$
(\mathrm{d} \beta)\left(g_{1}, \ldots, g_{p}\right)=(-1)^{p} \mathrm{~d}_{R h} \beta\left(g_{1}, \ldots, g_{p}\right),
$$

where $\mathrm{d}_{R h}$ is the de Rham differential, and

$$
\begin{aligned}
(\delta \beta)\left(g_{1}, \ldots, g_{p+1}\right)= & \beta\left(g_{2}, \ldots, g_{p+1}\right)+\sum_{i=1}^{p}(-1)^{i} \beta\left(g_{1}, \ldots, g_{i} g_{i+1}, \ldots, g_{p+1}\right) \\
& +(-1)^{p+1} L\left(g_{p+1}\right)^{*} \beta\left(g_{1}, \ldots, g_{p}\right)
\end{aligned}
$$

where $L(a): G / K \rightarrow G / K$ is the action of $a \in G$. (For $p=0$, this is to be interpreted as $(\delta \beta)\left(g_{1}\right)=\beta-L\left(g_{1}\right)^{*} \beta$.) The horizontal augmentation map $\mathrm{i}$ is simply the inclusion of the invariant forms (16), while $\mathrm{j}$ is the pullback of $V$-valued functions on $G^{p}$ under the map $G / K \rightarrow$ pt.

Suppose now that $G$ has finitely many components and that $K$ is a maximal compact subgroup of $G$. Recall that maximal compact subgroups are unique up to conjugation, and that the homogeneous space $G / K$ is diffeomorphic to the vector space $\mathfrak{g} / \mathfrak{k}$ (see, e.g., Borel [2, Chapter VII]). For $G$ semisimple, there is a canonical such diffeomorphism, by the Cartan decomposition $G=K \exp (\mathfrak{p})$.

Under the diffeomorphism $G / K \cong \mathfrak{g} / \mathfrak{k}$, the scalar multiplication of $\mathfrak{g} / \mathfrak{k}$ translates into a smooth deformation retraction

$$
\lambda:[0,1] \times G / K \rightarrow G / K, \quad(t, g K) \mapsto \lambda_{t}(g K),
$$

with $\lambda_{t_{1} t_{2}}=\lambda_{t_{1}} \circ \lambda_{t_{2}}$, interpolating between the identity map and the map $\iota_{e K} \circ \mathrm{pr}_{e K}$ where

$$
\operatorname{pr}_{e K}: G / K \rightarrow \mathrm{pt}, \quad \iota_{e K}: \mathrm{pt} \rightarrow G / K
$$

are projection to and inclusion of the base point $e K \in G / K$. It determines a de Rham homotopy operator on $V$-valued forms,

$$
T: \Omega^{q}(G / K, V) \rightarrow \Omega^{q-1}(G / K, V),
$$

given by pullback under (20), followed by integration over $[0,1]$. Thus $\left[\mathrm{d}_{R h}, T\right]=\mathrm{id}-\mathrm{pr}_{e K}^{*} \circ \iota_{e K}^{*}$. The homotopy operator has the properties $T \circ T=0$, as well as

$$
\left.(T \beta)\right|_{e K}=0
$$

for all $\beta \in \Omega^{q}(G / K, V)$.

$$
(\mathrm{k} \beta)\left(g_{1}, \ldots, g_{p}\right)=(-1)^{p} T\left(\beta\left(g_{1}, \ldots, g_{p}\right)\right) .
$$

Thus $[k, d]=1-\mathrm{j} \circ \mathrm{q}$ where

$$
(\mathbf{q} \beta)\left(g_{1}, \ldots, g_{p}\right)=\iota_{e K}^{*} \beta\left(g_{1}, \ldots, g_{p}\right) .
$$


The vertical homotopy operator determines a 'van Est integration map'

$$
R_{G / K}=\mathrm{q} \circ(1+\delta \mathrm{k})^{-1} \circ \mathrm{i}: \mathrm{C}^{\bullet}(\mathfrak{g}, K, V) \rightarrow \mathrm{C}^{\bullet}(G, V) .
$$

Proposition 3.4. The van Est integration map $R_{G / K}$ is a right inverse to the van Est differentiation map $\mathrm{VE}_{G / K}$.

Proof. By Lemma 2.3, it suffices to show that $\mathrm{h} \circ \mathrm{k}=0$ and $\mathrm{p} \circ \mathrm{k}=0$. Given $\beta$ as in (17), let $\psi: G^{p} \times G \rightarrow V \otimes\left(\wedge^{q} \mathfrak{g}^{*}\right)_{\mathfrak{k}-\text { hor }}$ be the corresponding $K$-equivariant map. The formula (11) shows that $\mathrm{h} \psi=0$ when $\left.\psi\right|_{G^{p} \times K}=0$. Consequently, in the differential form picture, $\mathrm{h} \beta=0$ whenever $\left.\beta\left(g_{1}, \ldots, g_{p}\right)\right|_{e K}=0$ for all $\left(g_{1}, \ldots, g_{p}\right) \in G^{p}$. In particular, this applies when $\beta$ is in the range of $T$. This shows $\mathrm{h} \circ \mathrm{k}=0$; the argument for $\mathrm{p} \circ \mathrm{k}=0$ is similar.

We will now give a more explicit description of $R_{G / K}$. For $\left(g_{1}, \ldots, g_{p}\right) \in G^{p}$ and $\left(t_{1}, \ldots, t_{p}\right) \in$ $[0,1]^{p}$ let

$$
\gamma_{t_{1}, \ldots, t_{p}}^{(p)}\left(g_{1}, \ldots, g_{p}\right)=\left(\lambda_{t_{1}} \circ L\left(g_{1}\right) \cdots \circ \lambda_{t_{p}} \circ L\left(g_{p}\right)\right)(e K) .
$$

For fixed $\left(g_{1}, \ldots, g_{p}\right)$, this defines a map $\gamma^{(p)}\left(g_{1}, \ldots, g_{p}\right):[0,1]^{p} \rightarrow G / K$.

Proposition 3.5. Given $\alpha \in \mathrm{C}^{p}(\mathfrak{g}, V)_{K \text {-basic }}$, let $\alpha_{G / K} \in \Omega^{p}(G / K, V)^{G}$ be the corresponding G-equivariant form. Then

$$
R_{G / K}(\alpha)\left(g_{1} \ldots, g_{p}\right)=\int_{[0,1]^{p}} \gamma^{(p)}\left(g_{1}, \ldots, g_{p}\right)^{*} \alpha_{G / K}
$$

Proof. We will calculate $R_{G / K}(\alpha)=(-1)^{p} \mathbf{q} \circ(\delta \mathbf{k})^{p}$ i $\alpha$ for $\alpha \in \mathrm{C}^{p}(\mathfrak{g}, V)$. We have that

$$
\mathrm{i} \alpha=\alpha_{G / K},
$$

viewed as an element of $\Omega^{p}(G / K, V)=\mathrm{D}^{0, p}(G, V)_{K \text {-basic }}$ Next, $\delta$ ki $\alpha \in \mathrm{D}^{1, p-1}(G, V)_{K \text {-basic }}$ is given by

$$
(\delta \operatorname{ki} \alpha)\left(g_{1}\right)=T \alpha_{G / K}-L\left(g_{1}\right)^{*} T \alpha_{G / K} .
$$

The next application of $\delta \mathbf{k}$ (or of $\mathbf{q}$, if $p=1$ ) will annihilate the first term, due to $T \circ T=0$ (respectively, due to $\left.\iota_{e K}^{*} \circ T=0\right)$. Hence we only need to keep the second term, and we find that $(\delta \mathrm{k})^{2} \mathrm{i} \alpha \in \mathrm{D}^{2, p-2}(G, V)_{K \text {-basic }}$ is given by

$\left((\delta \mathbf{k})^{2} \mathbf{i} \alpha\right)\left(g_{1}, g_{2}\right)=T \circ L\left(g_{2}\right)^{*} \circ T \alpha_{G / K}-T \circ L\left(g_{1} g_{2}\right)^{*} \circ T \alpha_{G / K}+L\left(g_{2}\right)^{*} \circ T \circ L\left(g_{1}\right)^{*} \circ T \alpha_{G / K}$.

By the same reasoning as before, we need only keep the last term, since all terms starting with $T$ will be annihilated by the subsequent application of $\mathrm{k}$ (respectively $\mathrm{q}$, if $p=2$ ). Proceeding in this manner, we arrive at the formula

$$
R_{G / K}(\alpha)\left(g_{1} \ldots, g_{p}\right)=\iota_{e K}^{*} \circ L\left(g_{p}\right)^{*} \circ T \circ \cdots \circ L\left(g_{1}\right)^{*} \circ T \alpha_{G / K} .
$$

(The $(-1)^{p}$ sign in the formula for $R_{G / K}$ is compensated by the alternating signs in the $L\left(g_{i}\right)^{*} \circ T$ contributions.) By definition, each $T$ involves pullback under the map $\lambda$, followed by integration over $[0,1]$. Denoting by $t_{i}$ the variable for the $i$-th such integration, and by $\lambda^{(i)}$ the map corresponding to $\lambda$ for the variable $t_{i}$, we arrive at

$$
R_{G / K}(\alpha)\left(g_{1} \ldots, g_{p}\right)=\int_{t_{p} \in[0,1]} \cdots \int_{t_{1} \in[0,1]} \iota_{e K}^{*} L\left(g_{p}\right)^{*}\left(\lambda^{(p)}\right)^{*} \cdots L\left(g_{1}\right)^{*}\left(\lambda^{(1)}\right)^{*} \alpha_{G / K}
$$


On the other hand, by definition,

$$
\lambda^{(1)} \circ L\left(g_{1}\right) \circ \cdots \lambda^{(p)} \circ L\left(g_{p}\right) \circ \iota_{e K}=\gamma^{(p)}\left(g_{1}, \ldots, g_{p}\right) .
$$

This gives (23) (with the orientation of $[0,1]^{p}$ given by the volume element $\mathrm{d} t_{p} \wedge \cdots \wedge \mathrm{d} t_{1}$ ).

In summary, we obtain the following cochain-level version of van Est's theorem:

Theorem 3.6 (Van Est theorem for Lie groups). Suppose $K$ is a maximal compact subgroup of the Lie group $G$. Then the van Est differentiation map

$$
\mathrm{VE}_{G / K}: \mathrm{C}^{\bullet}(G, V) \rightarrow \mathrm{C}^{\bullet}(\mathfrak{g}, V)_{K-\text { basic }}
$$

defined by (15) is a homotopy equivalence. The van Est integration map

$$
R_{G / K}: \mathrm{C}^{\bullet}(\mathfrak{g}, V)_{K-\text { basic }} \rightarrow \mathrm{C}^{\bullet}(G, V)
$$

given by (23) is a right inverse at the level of cochains.

Remark 3.7. Sometimes, it is convenient to work with the normalized subcomplex $\widetilde{\mathrm{C}}(G, V)$, consisting of functions $f \in C^{\infty}\left(G^{p}, V\right)$ with the property that $f\left(g_{1}, \ldots, g_{p}\right)=0$ whenever $g_{i}=e$ for some $i$.The inclusion of the normalized subcomplex is well-known to be a homotopy equivalence (see, e.g., [21]). Theorem 3.6 holds for the normalized subcomplex, with the same proof.

If $K$ is any compact Lie subgroup of $G$ (not necessarily maximal compact), one obtains a similar conclusion by replacing $\mathrm{C}(G, V)$ with the localized complex

$$
\mathrm{C}(G, V)_{e}=\mathrm{C}(G, V) / \sim
$$

of germs of functions $G^{p} \rightarrow V$ at $(e, \ldots, e) \in G^{p}$, and using a germ at $e K$ of a diffeomorphism $G / K \rightarrow \mathfrak{g} / \mathfrak{k}$ to define a germ of a retraction $\lambda_{t}$. One hence obtains a homotopy equivalence

$$
\mathrm{VE}_{G / K}: \mathrm{C}^{\bullet}(G, V)_{e} \rightarrow \mathrm{C}^{\bullet}(\mathfrak{g}, V)_{K-\text { basic }}
$$

with a homotopy inverse $R_{G / K}$ which is also a right inverse. In particular, this is true for $K=\{e\}$, cf. [16, 25].

\section{VAN Est THEORY FOR LIE GROUPOIDS}

We will next review the cochain complexes for Lie algebroids and Lie groupoids, and the van Est double complex connecting them. We then show how certain horizontal and vertical homotopy operators on the double complex define van Est differentiation and integration maps, and finally show that the integration map is right inverse to the differentiation. Only at the end, we will derive the 'explicit formulas' for the integration and differentiation. For basic information on Lie groupoids and Lie algebroids, we refer to [8, 10, 20]. The van Est map for

Lie groupoids was introduced by Weinstein-Xu in 30 and further studied by Crainic [7; for further generalizations and applications see, [1, 5, 6, 18, 19, 22]. 
4.1. The simplicial manifold $B_{p} G$. Let $G \rightrightarrows M$ be a Lie groupoid, with source and target maps denoted s, t: $G \rightarrow M$. Elements $g, h \in G$ are composable if $\mathrm{s}(g)=\mathrm{t}(h)$; in this case their groupoid product is denoted as $g h$. We denote by

$$
B_{p} G=\left\{\left(g_{1}, \ldots, g_{p}\right) \mid \mathrm{s}\left(g_{i}\right)=\mathrm{t}\left(g_{i+1}\right), \quad 0<i<p\right\}
$$

the space of $p$-arrows; by convention $B_{0} G=M$. Every $p$-arrow comes with $p+1$ base points $\left(m_{0}, \ldots, m_{p}\right)$, where $m_{i}=\mathrm{s}\left(g_{i}\right)=\mathrm{t}\left(g_{i+1}\right)$. The collection of spaces $B_{p} G$ defines a simplicial manifold $B_{\bullet} G$ called the nerve of the groupoid. The face map $\partial_{i}: B_{p} G \rightarrow B_{p-1} G$ drops the $i$-th base point:

$$
\partial_{i}\left(g_{1}, \ldots, g_{p}\right)= \begin{cases}\left(g_{2}, \ldots, g_{p}\right), & i=0, \\ \left(g_{1}, \ldots, g_{i} g_{i+1}, \ldots, g_{p}\right), & 0<i<p, \\ \left(g_{1}, \ldots, g_{p-1}\right), & i=p,\end{cases}
$$

while the $i$-th degeneracy map $\epsilon_{i}: B_{p} G \rightarrow B_{p+1} G$ repeats the $i$-th base point by inserting a trivial arrow:

$$
\epsilon_{i}\left(g_{1}, \ldots, g_{p}\right)=\left(g_{1}, \ldots, g_{i}, m_{i}, g_{i+1}, \ldots, g_{p}\right), \quad i=0, \ldots, p .
$$

Given a $G$-action on manifold $Q$, with anchor $\Phi: Q \rightarrow M$, one obtains a simplicial manifold

$$
B_{p} G \times{ }_{M} Q
$$

where the fiber product is with respect to $\Phi$ and the map taking the $p$-arrow $\left(g_{1}, \ldots, g_{p}\right) \in B_{p} G$ to the base point $m_{p}$. The face and degeneracy maps maps are

$$
\begin{aligned}
\partial_{i}\left(g_{1}, \ldots, g_{p} ; x\right), & = \begin{cases}\left(g_{2}, \ldots, g_{p} ; x\right), & i=0, \\
\left(g_{1}, \ldots, g_{i} g_{i+1}, \ldots, g_{p} ; x\right), & 0<i<p, \\
\left(g_{1}, \ldots, g_{p-1} ; g_{p} x\right), & i=p,\end{cases} \\
\epsilon_{i}\left(g_{1}, \ldots, g_{p} ; x\right) & =\left(g_{1}, \ldots, g_{i}, m_{i}, g_{i+1}, \ldots, g_{p} ; x\right), \quad 0 \leq i \leq p .
\end{aligned}
$$

(More conceptually, these formulas are explained through the identification $B_{p} G \times_{M} Q \cong$ $B_{p}(G \ltimes Q)$, where $G \ltimes Q \rightrightarrows Q$ is the action groupoid.) The manifolds $B_{p} G \times_{M} Q$ come equipped with $p+1$ commuting $G$-actions (cf. (13)):

$$
a \cdot\left(g_{1}, \ldots, g_{p} ; x\right)= \begin{cases}\left(a g_{1}, g_{2}, \ldots, g_{p} ; x\right) & i=0, \\ \left(g_{1}, \ldots, g_{i} a^{-1}, a g_{i+1}, \ldots, g_{p} ; x\right) & 0<i<p, \\ \left(g_{1}, \ldots, g_{p-1}, g_{p} a^{-1} ; a \cdot x\right) & i=p .\end{cases}
$$

with anchor map $\left(g_{1}, \ldots, g_{p} ; x\right) \mapsto m_{i}$.

\subsection{The van Est double complex.}

4.2.1. Groupoid complex. Given a representation of the groupoid $G \rightrightarrows M$ on a vector bundle $V \rightarrow M$, taking $Q=V$ in (24), we obtain a simplicial vector bundle $B_{p} G \times_{M} V \rightarrow B_{p} G$. The groupoid cochain complex $(\mathrm{C}(G, V), \delta)$ has graded components

$$
\mathrm{C}^{p}(G, V)=\Gamma\left(B_{p} G \times_{M} V\right),
$$

while the differential $\delta$ is given on $p$-cochains by $\delta=\sum_{i=0}^{p+1}(-1)^{i} \partial_{i}^{*}$. In the case of trivial coefficients $V=M \times \mathbb{R}$, we write $\mathrm{C}(G)=\mathrm{C}(G, M \times \mathbb{R})$. 
4.2.2. Lie algebroid complex. Let $A \Rightarrow M$ be the Lie algebroid of $G \rightrightarrows M$. Thus, $A$ is the vector bundle whose sections are the left-invariant vector fields on $G$ (tangent to the t-fibers); for $\xi \in \Gamma(A)$ we denote by $\xi^{L}$ the corresponding left-invariant vector field. The anchor map a: $A \rightarrow$ $T M$ is characterized by its property $\xi^{L} \sim_{\mathrm{s}}-\mathrm{a}(\xi)$. The $G$-representation on $V$ determines an $A$-representation on $V$. (Every $\xi \in \Gamma(A)$ is realized as the derivative of a 1-parameter family of bisections of $G$. The group of bisections acts linearly on the sections of $V$, and by differentiation one obtains the flat $A$-connection $\xi \mapsto \nabla_{\xi}$ defining the $A$-representation.) Let $\left(\mathrm{C}(A, V), \mathrm{d}_{C E}\right)$ be the resulting Lie algebroid complex (or Chevalley-Eilenberg complex), with p-cochains

$$
\mathrm{C}^{p}(A, V)=\Gamma\left(V \otimes \wedge^{p} A^{*}\right),
$$

and with the Chevalley-Eilenberg differential $\mathrm{d}_{C E}$. For $\xi \in \Gamma(A)$, we denote by $\iota_{\xi}$ the operator on $\mathrm{C}(A, V)$ given by contraction, and by $\mathcal{L}_{\xi}=\left[\mathrm{d}_{C E}, \iota_{\xi}\right]$ the Lie derivative. On $\mathrm{C}^{0}(A, V)=\Gamma(V)$, we have that $\mathcal{L}_{\xi}=\nabla_{\xi}$. In the case of the trivial representation on $V=M \times \mathbb{R}$, the connection is $\nabla_{\xi}=\mathcal{L}_{\mathrm{a}(\xi)}$ where a: $A \rightarrow T M$ is the anchor of $A$; we will write $\mathrm{C}(A)=\mathrm{C}(A, M \times \mathbb{R})$.

4.2.3. Double complex. The two complexes $\mathrm{X}=\mathrm{C}(A, V), \mathrm{Y}=\mathrm{C}(G, V)$ are related by a van Est double complex, due to Crainic [7]. Taking $Q=G, \Phi=\mathrm{t}$ in (24), with the $G$-action by left multiplication, define a simplicial fiber bundle

$$
\kappa_{p}: E_{p} G=B_{p} G \times{ }_{M} G \rightarrow B_{p} G .
$$

For each $p$ this is a principal $G$-bundle, with anchor map

$$
\pi_{p}\left(g_{1}, \ldots, g_{p} ; g\right)=\mathrm{s}(g),
$$

and principal action

$$
a \cdot\left(g_{1}, \ldots, g_{p} ; g\right)=\left(g_{1}, \ldots, g_{p} ; g a^{-1}\right) .
$$

(For background on principal bundles for Lie groupoids, see for example [20.) Each of these principal bundles is actually trivial, with a trivializing section

$$
\nu_{p}: B_{p} G \rightarrow E_{p} G, \quad\left(g_{1}, \ldots, g_{p}\right) \mapsto\left(g_{1}, \ldots, g_{p} ; m\right)
$$

where $m=\mathrm{s}\left(g_{p}\right)$.

The face and degeneracy maps are principal bundle morphisms, making $\kappa: E G \rightarrow B G$ into a simplicial principal bundle; the 'universal bundle' of the Lie groupoid $G$. (Note that (27) are not simplicial maps, and indeed $E G$ is non-trivial as a simplicial principal bundle.) The van Est double complex

$$
\left(\mathrm{D}^{\bullet \bullet \bullet}(G, V), \delta, \mathrm{d}\right)
$$

is defined as follows.

- The bigraded summands of the double complex are

$$
\mathrm{D}^{p, q}(G, V)=\Gamma\left(\pi_{p}^{*}\left(V \otimes \wedge^{q} A^{*}\right)\right),
$$

generalizing the description (9) in the case of Lie groups.

- $\delta$ is the simplicial differential on sections of the simplicial vector bundle

$$
\pi_{\bullet}^{*}\left(\wedge^{q} A^{*} \otimes V\right) \rightarrow E_{\bullet} G .
$$


- $\mathrm{d}=(-1)^{p} \mathrm{~d}_{C E}$ on elements of bidegree $(p, q)$, with the Chevalley-Eilenberg differential on

$$
\mathrm{D}^{p, \bullet}(G, V) \cong \mathrm{C}^{\bullet}\left(\pi_{p}^{*} A, \pi_{p}^{*} V\right)
$$

In more detail, let $\mathcal{F}$ be the foliation of $E_{p} G$ given by the $\kappa_{p}$-fibers; thus $T_{\mathcal{F}} E_{p} G$ is the vertical bundle. The isomorphism $\pi_{p}^{*} A \cong T_{\mathcal{F}} E_{p} G$ defines a Lie algebroid structure on $\pi_{p}^{*} A$. On the other hand, the isomorphism $\mathrm{t}^{*} V \cong \mathrm{s}^{*} V$ given by the $G$-representation extends to an isomorphism, for any $p$,

$$
\kappa_{p}^{*}\left(B_{p} G \times_{M} V\right) \cong \pi_{p}^{*} V .
$$

Since this bundle is trivial along the leaves of $\mathcal{F}$, it comes with a natural representation of the Lie algebroid $\pi_{p}^{*} A=T_{\mathcal{F}} E_{p} G$, and the right hand side of (29) is its Lie algebroid complex.

Furthermore, the double complex comes with horizontal and vertical augmentation maps:

- $\mathrm{i}: \mathrm{C}^{\bullet}(A, V) \rightarrow \mathrm{D}^{0, \bullet}(G, V)$ is given in degree $q$ by the pullback $\pi_{0}^{*}$ (using (29) for $p=0$ ).

- $\mathrm{j}: \mathrm{C}^{\bullet}(G, V) \rightarrow \mathrm{D}^{\bullet}, 0(G, V)$ is given in degree $p$ by the pullback map $\kappa_{p}^{*}$ (using (30) ).

The augmentation maps define cochain maps to the total complex

$$
\mathrm{C}^{\bullet}(G, V) \stackrel{\mathrm{j}}{\longrightarrow} \operatorname{Tot}^{\bullet}(\mathrm{D}(G, V)) \stackrel{\mathrm{i}}{\longleftarrow} \mathrm{C}^{\bullet}(A, V)
$$

Remark 4.1. Sometimes, it is better to work with the normalized subcomplex, defined by the requirement that all pull-backs under the degeneracy maps $\epsilon_{i}$ are equal to zero. We will indicate the normalized subcomplexes (and the spaces of functions and sections defining them) by a tilde; for example

$$
\widetilde{\mathrm{C}}^{\bullet}(G, V)=\widetilde{\Gamma}\left(B \bullet G \times_{M} V\right), \quad \widetilde{\mathrm{D}}^{\bullet}, \bullet(G, V)=\widetilde{\Gamma}\left(\pi_{p}^{*}\left(V \otimes \wedge^{q} A^{*}\right)\right) .
$$

By a general result for simplicial manifolds (see e.g. [21]), the inclusion $\widetilde{\mathrm{C}}(G, V) \hookrightarrow \mathrm{C}(G, V)$ is a homotopy equivalence.

As another variation, we will consider localized versions of these complexes, with respect to the submanifold $M \subseteq B_{p} G$ of constant $p$-arrows. These cochain complexes

$$
C^{\bullet}(G, V)_{M}=\Gamma\left(B \bullet G \times{ }_{M} V\right)_{M},
$$

are given by germs of sections along the submanifold $M \subseteq B_{\bullet} G$. There is also a localized version $\mathrm{D}^{\bullet \bullet} \bullet(G, V)_{M}$ of the double complex (and its normalized subcomplex), working with germs of sections along $M \subseteq E_{\bullet} G$. Note that the localized version (as well as its normalized subcomplex) also makes sense for local Lie groupoids.

\subsection{Differentiation.}

4.3.1. The horizontal homotopy. The simplicial universal bundle EG comes with a simplicial retraction onto its submanifold $M$ (see [23] and [18, Appendix A.2]). This is reflected in the existence of a homotopy operator on the double complex $\mathrm{D}(G, V)$. Consider the maps

$$
h_{p}: E_{p} G \rightarrow E_{p+1} G,\left(g_{1}, \ldots, g_{p} ; g\right) \mapsto\left(g_{1}, \ldots, g_{p}, g ; m\right)
$$

where $m=\mathrm{s}(g)$. Since $\pi_{p+1} \circ h_{p}=\pi_{p}$, these lift to fiberwise isomorphisms of vector bundles $\pi_{p}^{*}\left(\wedge^{q} A^{*} \otimes V\right) \rightarrow \pi_{p+1}^{*}\left(\wedge^{q} A^{*} \otimes V\right)$, defining a pullback map on sections. 
Lemma 4.2. The map

$$
\mathrm{h}: \mathrm{D}^{p, q}(G, V) \rightarrow \mathrm{D}^{p-1, q}(G, V), \quad \psi \mapsto(-1)^{p} h_{p-1}^{*} \psi
$$

satisfies

$$
[\mathrm{h}, \delta]=1-\mathrm{i} \circ \mathrm{p},
$$

where $\mathrm{p}: \mathrm{D}^{0, \bullet}(G, V) \rightarrow \mathrm{C}^{\bullet}(A, V)$ is the left inverse to $\mathrm{i}=\pi_{0}^{*}$ given by pullback under the inclusion $u: M \hookrightarrow E_{0} G=G$ :

$$
\mathrm{p}=u^{*}: \Gamma\left(\pi_{0}^{*}\left(\wedge^{q} A^{*} \otimes V\right)\right) \rightarrow \Gamma\left(\wedge^{q} A^{*} \otimes V\right) .
$$

Proof. Let $\psi \in \mathrm{D}^{p, q}(G, V)$. If $p=0$ we have that $\mathrm{h} \psi=0$, while $(\delta \psi)\left(g_{1} ; g\right)=\psi(g)-$ $\psi\left(g_{1} g\right)$ and therefore $(h \delta \psi)(g)=-(\delta \psi)(g ; m)=\psi(g)-\psi(m)$. For $p<0$ we have that $(\mathrm{h} \psi)\left(g_{1}, \ldots, g_{p-1} ; g\right)=(-1)^{p} \psi\left(g_{1}, \ldots, g_{p-1}, g ; m\right)$ and therefore

$$
\begin{aligned}
(\delta \mathrm{h} \psi)\left(g_{1}, \ldots, g_{p} ; g\right)= & (-1)^{p}\left(\psi\left(g_{2}, \ldots, g_{p} ; m\right)-\psi\left(g_{1} g_{2}, \ldots, g_{p}, g ; m\right) \pm \cdots\right. \\
& \left.\left.+(-1)^{p} \psi\left(g_{1}, \ldots, g_{p-1}, g ; m\right)\right)\right)
\end{aligned}
$$

Similarly,

$$
\begin{aligned}
(\mathrm{h} \delta \psi)\left(g_{1}, \ldots, g_{p} ; g\right)= & (-1)^{p+1}\left(\psi\left(g_{2}, \ldots, g_{p} ; m\right)-\psi\left(g_{1} g_{2}, \ldots, g_{p}, g ; m\right) \pm \cdots\right. \\
& \left.\left.+(-1)^{p+1} \psi\left(g_{1}, \ldots, g_{p} ; g\right)\right)\right) .
\end{aligned}
$$

Adding the two expressions, all terms except for $\psi\left(g_{1}, \ldots, g_{p} ; m\right)$ cancel.

Note that since $\kappa_{p+1} \circ h_{p} \neq \kappa_{p}$, in general, the maps $h_{p}$ need not preserve the foliation $\mathcal{F}$, and hence the homotopy operator $h$ and the projection $\mathrm{p}$ do not usually commute with the differential $d$.

Remark 4.3. The homotopy operator $\mathrm{h}$ and the projection $\mathrm{p}$ restrict to the normalized subcomplex $\widetilde{D}^{\bullet} \bullet(G, V)$. On this subcomplex, they have the additional properties

$$
\mathrm{h} \circ \mathrm{h}=0, \quad \mathrm{p} \circ \mathrm{h}=0
$$

this follows because $h_{p}$ coincides on the range of $h_{p-1}$ (or of $u: M \hookrightarrow G_{0}$, in case $p=0$ ) with the degeneracy map $\epsilon_{p}$.

4.3.2. Van Est map. The Perturbation Lemma 2.1 gives a new projection $\mathrm{p}^{\prime}=\mathrm{p} \circ(1+\mathrm{dh})^{-1}$, which is a cochain map for the total differential $d+\delta$, with $\mathrm{p}^{\prime} \circ \mathrm{i}=\mathrm{id}$. Thus, $\mathrm{i}$ is a homotopy equivalence, with $p^{\prime}$ a homotopy inverse. We obtain a cochain map

$$
\mathrm{VE}_{G}=\mathrm{p} \circ(1+\mathrm{dh})^{-1} \circ \mathrm{j}: \mathrm{C}^{\bullet}(G, V) \rightarrow \mathrm{C}^{\bullet}(A, V) .
$$

For a more explicit description of this map, recall the commuting $G$-actions (25) on $B_{p} G \times{ }_{M} Q$, for a $G$-manifold $Q$. These actions have generating vector fields $\xi^{(i)}, \xi \in \Gamma(A), i=1, \ldots, p$. In the case of $Q=V$, the $\xi^{(i)}$ are linear with respect to the vector bundle structure on $B_{p} G \times_{M} V \rightarrow B_{p} G$. They hence define covariant derivatives

$$
\nabla_{\xi}^{(i)}: \Gamma\left(B_{p} G \times_{M} V\right) \rightarrow \Gamma\left(B_{p} G \times_{M} V\right) .
$$


Theorem 4.4. [18] The map $\mathrm{VE}_{G}$ is given by the formula,

$$
\mathrm{VE}_{G}(\sigma)\left(\xi_{1}, \ldots, \xi_{p}\right)=\left.\sum_{s \in \mathfrak{S}_{p}} \operatorname{sign}(s) \nabla_{\xi_{s(1)}}^{(1)} \cdots \nabla_{\xi_{s(p)}}^{(p)}(\sigma)\right|_{M}
$$

for $\sigma \in \mathrm{C}^{p}(G, V)=\Gamma\left(B_{p} G \times_{M} V\right)$ and $\xi_{1}, \ldots, \xi_{p} \in \Gamma(A)$. Here the sum is over the permutation group $\mathfrak{S}_{p}$, and $M$ is regarded as a submanifold of $B_{p} G$ consisting of constant p-arrows.

Remark 4.5. Equation (33) is Weinstein-Xu's formula [30] for the van Est map $\mathrm{VE}_{G}$. To be precise, 30 only treated the case of trivial coefficients, and exclusively worked with the normalized subcomplex $\widetilde{\mathrm{C}}(G)$. They proved by direct computation that this expression defines a cochain map, and furthermore that it intertwines the cup product on groupoid cochains with the wedge product on Lie algebroid cochains. The latter fact only holds true on the normalized subcomplex. In [18, it was explained by additional properties of the homotopy operator on the normalized sub-double complex, such as (31).

We include a proof of Theorem 4.4 in the Appendix. (It is a slightly simplified version of the argument in [18].)

4.4. Integration. We next discuss the integration from Lie algebroid cochains to Lie groupoid cochains. We will work with the localized complex $C^{\bullet}(G, V)_{M}$ defined in terms of germs of sections along $M \subseteq B_{p} G$; here $G$ could also be only a local Lie groupoid. For convenience, we will typically omit explicit emphasis of 'germs' and 'local'. If $G$ is a Lie groupoid which happens to be globally contractible to $M$ along its t-fibers, one may work with the complex $\mathrm{C}(G, V)$.

4.4.1. Differential form picture of double complex. Just as in the case that $G$ is a Lie group, the discussion of integration is more convenient using an interpretation in term of differential forms. Let $\left(\Omega_{\mathcal{F}}\left(G, \mathrm{t}^{*} V\right), \mathrm{d}_{R h}\right)$ be the de Rham complex of foliated (leafwise) $\mathrm{t}^{*} V$-valued forms on $G$. Restriction to $M \subseteq G$ takes such a form to a section of $V \otimes \wedge A^{*}$, and induced an isomorphism of differential complexes,

$$
\left(\Omega_{\mathcal{F}}^{\bullet}\left(G, \mathrm{t}^{*} V\right)^{L}, \mathrm{~d}_{R h}\right) \cong\left(\mathrm{C}^{\bullet}(A, V), \mathrm{d}_{C E}\right) .
$$

Observe furthermore that

$$
\begin{aligned}
& \pi_{p}^{*} A=T_{\mathcal{F}} E_{p} G=B_{p} G \times_{M} T_{\mathcal{F}} G, \\
& \pi_{p}^{*} V=\kappa_{p}^{*}\left(B_{p} G \times_{M} V\right)=B_{p} G \times_{M} \mathrm{t}^{*} V .
\end{aligned}
$$

We may hence regard the elements of $\mathrm{D}^{p, q}(G, V)$ as maps

$$
\beta: B_{p} G \rightarrow \Omega_{\mathcal{F}}^{q}\left(G, \mathrm{t}^{*} V\right),\left(g_{1}, \ldots, g_{p}\right) \mapsto \beta\left(g_{1}, \ldots, g_{p}\right)
$$

such that $\beta\left(g_{1}, \ldots, g_{p}\right) \in \Omega^{q}\left(\mathrm{t}^{-1}(m)\right) \otimes V_{m}$ for $m=\mathbf{s}\left(g_{p}\right)$, and smoothly depending on $\left(g_{1}, \ldots, g_{p}\right)$. Similarly, $\mathrm{D}^{p, q}(G, V)_{M}$ is interpreted as germs along $M \subseteq B_{p} G$ of such maps.

In this picture, the vertical differential is $\mathrm{d}=(-1)^{p} \mathrm{~d}_{R h}$ (cf. (18)), while the horizontal differential is described similar to (19). The augmentation map $i$ is the inclusion of (34),

$$
\mathrm{i}: \Omega_{\mathcal{F}}^{q}\left(G, \mathrm{t}^{*} V\right)^{L} \hookrightarrow \mathrm{D}^{0, q}(G, V)=\Omega_{\mathcal{F}}^{q}\left(G, \mathrm{t}^{*} V\right)
$$

while $\mathrm{j}$ is the inclusion of $\Gamma\left(B_{p} G \times{ }_{M} V\right)$ into the space of maps $\beta: B_{p} G \rightarrow \Omega_{\mathcal{F}}^{0}\left(G, \mathrm{t}^{*} V\right)$ such that $\beta\left(g_{1}, \ldots, g_{p}\right) \in C^{\infty}\left(\mathrm{t}^{-1}(m)\right) \otimes V_{m}$ is constant on $\mathrm{t}^{-1}(m)$, for any given $\left(g_{1}, \ldots, g_{p}\right)$ with $m=\mathrm{s}\left(g_{p}\right)$. 
4.4.2. The integration map $R_{G}$. A tubular structure for a (local) Lie groupoid $G \rightrightarrows M$ is a tubular neighborhood embedding

$$
\left.A \cong \operatorname{ker}(T \mathrm{t})\right|_{M} \rightarrow G
$$

taking the fibers of $A \rightarrow M$ to the t-fibers, and with differential along $M$ the identity map of $A$. The tubular structure transports the scalar multiplication in $A$ to a retraction along t-fibers

$$
\lambda:[0,1] \times G \rightarrow G,(t, g) \mapsto \lambda_{t}(g),
$$

or more precisely the germ along $[0,1] \times M$ of such a map. Here $\lambda_{0}=u \circ \mathrm{t}$, where $u: M \rightarrow G$ is the inclusion of units. The retraction determines a homotopy operator

$$
T: \Omega_{\mathcal{F}}^{q}\left(G, \mathrm{t}^{*} V\right)_{M} \rightarrow \Omega_{\mathcal{F}}^{q-1}\left(G, \mathrm{t}^{*} V\right)_{M}
$$

given by pullback under $\lambda$ followed by integration over $[0,1]$. This has the properties $T \circ T=0$ and

$$
\left.T \beta\right|_{M}=0 .
$$

Similar to (21), it defines a vertical homotopy operator $k$ on the double complex, where

$$
(\mathrm{k} \beta)\left(g_{1}, \ldots, g_{p}\right)=(-1)^{p} T\left(\beta\left(g_{1}, \ldots, g_{p}\right)\right)
$$

That is, $[\mathrm{k}, \mathrm{d}]=1-\mathrm{j} \circ \mathrm{q}$ where, for $\beta$ of bidegree $(p, 0)$,

$$
(\mathbf{q} \beta)\left(g_{1}, \ldots, g_{p}\right)=u^{*} \beta\left(g_{1}, \ldots, g_{p}\right)
$$

(the restriction of $\beta\left(g_{1}, \ldots, g_{p}\right) \in \Gamma\left(\mathrm{t}^{*} V\right)$ to the units). The properties of $T$ show that

$$
\mathrm{k} \circ \mathrm{k}=0, \quad \mathrm{q} \circ \mathrm{k}=0 .
$$

By the Perturbation Lemma 2.1, we obtain a cochain map

$$
R_{G}=\mathrm{q} \circ(1+\delta \mathrm{k})^{-1} \circ \mathrm{i}: \mathrm{C}^{\bullet}(A, V) \rightarrow \mathrm{C}^{\bullet}(G, V)_{M} .
$$

Note again that on elements of degree $p$, the map $R_{G}$ is given by a zig-zag $(-1)^{p} \mathbf{q} \circ(\delta \mathrm{k})^{p} \circ \mathrm{i}$.

Remark 4.6. The vertical homotopy k restricts to the normalized sub-double complex $\widetilde{\mathrm{D}}(G, V)_{M}$. Hence, $R_{G}$ takes values in the normalized subcomplex $\widetilde{\mathrm{C}}(G, V)_{M}$.

Proposition 4.7. The integration map $R_{G}$ is right inverse to the van Est differentiation $\mathrm{VE}_{G}$ :

$$
\mathrm{VE}_{G} \circ R_{G}=\operatorname{id}_{\mathrm{C}(A, V)} \text {. }
$$

Proof. Let $\psi \in \mathrm{D}^{p, q}(G, V)$ with the corresponding map $\beta: B_{p} G \rightarrow \Omega_{\mathcal{F}}^{q}\left(G, \mathrm{t}^{*} V\right)$. By the properties of $T$,

$$
\left.(\mathrm{k} \beta)\left(g_{1}, \ldots, g_{p}\right)\right|_{M}=0 .
$$

This means that the section $\mathrm{k} \psi \in \Gamma\left(\pi_{p}^{*}\left(V \otimes \wedge^{q-1} A^{*}\right)\right)$ corresponding to $\mathrm{k} \beta$ vanishes along $B_{p} G \times_{M} M \subseteq E_{p} G=B_{p} G \times_{M} G$. But then $\mathrm{hk} \psi=(-1)^{p} h_{p-1}^{*} \mathrm{k} \psi=0$. This shows $\mathrm{h} \circ \mathrm{k}=0$; similarly we obtain $\mathrm{p} \circ \mathrm{k}=0$. Now use Lemma 2.3 . 
4.4.3. A formula for $R_{G}$. We will now show that the van Est integration map $R_{G}$ coincides with the map defined in [6]. Define a a map $[0,1]^{p} \times B_{p} G \rightarrow G$ (more precisely, a germ along $[0,1]^{p} \times M$ of such a map) by the formula:

$$
\gamma_{t_{1}, \ldots, t_{p}}^{(p)}\left(g_{1}, \ldots, g_{p}\right)=\lambda_{t_{1}}\left(g_{1} \lambda_{t_{2}}\left(g_{2} \cdots \lambda_{t_{p}}\left(g_{p}\right) \cdots\right)\right)
$$

For fixed $\left(g_{1}, \ldots, g_{p}\right)$ (close to $\left.M\right)$, this is a smooth map from the unit cube $[0,1]^{p}$ into the t-fiber of $m_{0}=\mathrm{t}\left(g_{1}\right)$.

Theorem 4.8. The van Est integration map $R_{G}=\mathrm{q} \circ(1+\delta \mathrm{k})^{-1} \circ \mathrm{i}$ is given on degree $p$ elements $\alpha \in \mathrm{C}^{p}(A, V)$ by the formula

$$
R_{G}(\alpha)\left(g_{1}, \ldots, g_{p}\right)=\int_{[0,1]^{p}}\left(\gamma^{(p)}\left(g_{1}, \ldots, g_{p}\right)\right)^{*}\left(\left.\alpha^{L}\right|_{\mathbf{t}^{-1}\left(m_{0}\right)}\right),
$$

Here $\alpha^{L} \in \Omega_{\mathcal{F}}^{p}\left(G, \mathrm{t}^{*} V\right)_{M}$ is the left-invariant foliated form defined by $\alpha \in \mathrm{C}^{p}(A, V)$. We think of $\alpha^{L}$ as a family of $V_{m}$-valued forms on the fibers $\mathrm{t}^{-1}(m)$; for fixed $\left(g_{1}, \ldots, g_{p}\right)$ the map $\gamma^{(p)}\left(g_{1}, \ldots, g_{p}\right)$ takes values in one such fiber, hence the pull-back is an ordinary form on $[0,1]^{p}$.

Remark 4.9. In [6], it was shown by direct calculation that the right hand side of (38) is a cochain map, which is a right inverse to $\mathrm{VE}_{G}$ at the level of cochains.

4.4.4. Proof of Theorem 4.8. The proof will require some preliminary results. Observe first the following alternative description of the maps $\gamma^{(p)}$. Denote by $\lambda_{t_{p}}^{(p)}$ the map $E_{p-1} G \rightarrow E_{p-1} G$ given by $\left(g_{1}, \ldots, g_{p-1}, g\right) \mapsto\left(g_{1}, \ldots, g_{p-1}, \lambda_{t_{p}}(g)\right)$.

Lemma 4.10. The map (37) is a composition

$$
\gamma_{t_{1}, \ldots, t_{p}}^{(p)}=\lambda_{t_{1}}^{(1)} \circ \partial_{1} \circ \lambda_{t_{2}}^{(2)} \circ \partial_{2} \circ \cdots \circ \lambda_{t_{p}}^{(p)} \circ \partial_{p} \circ \nu_{p}: B_{p} G \rightarrow G .
$$

Proof. By direct calculation,

$$
\begin{aligned}
\left(g_{1}, \ldots, g_{p}\right) & \stackrel{\nu_{p}}{\longmapsto}\left(g_{1}, \ldots, g_{p} ; \mathrm{s}\left(g_{p}\right)\right) \\
& \stackrel{\partial_{p}}{\longmapsto}\left(g_{1}, \ldots, g_{p-1} ; g_{p}\right) \\
& \stackrel{\lambda_{t_{p}}^{(p)}}{\longmapsto}\left(g_{1}, \ldots, g_{p-1} ; \lambda_{t_{p}}\left(g_{p}\right)\right) \\
& \stackrel{\partial_{p-1}}{\longmapsto}\left(g_{1}, \ldots, g_{p-2} ; g_{p-1} \lambda_{t_{p}}\left(g_{p}\right)\right) \\
& \stackrel{\lambda_{t_{p-1}}^{(p-1)}}{\longmapsto}\left(g_{1}, \ldots, g_{p-2} ; \lambda_{t_{p-1}}\left(g_{p-1} \lambda_{t_{p}}\left(g_{p}\right)\right)\right) \\
& \ldots
\end{aligned}
$$

eventually arriving at (37).

We will also need:

Lemma 4.11. For $0 \leq i \leq p$ (but usually not for $i=p+1$ ), we have that

$$
\begin{aligned}
& \partial_{i}^{*} \circ \mathrm{k}=\mathrm{k} \circ \partial_{i}^{*}: \mathrm{D}^{p, q}(G, V)_{M} \rightarrow \mathrm{D}^{p+1, q-1}(G, V)_{M}, \\
& \partial_{i}^{*} \circ \mathrm{q}=\mathrm{q} \circ \partial_{i}^{*}: \mathrm{D}^{p, 0}(G, V)_{M} \rightarrow \mathrm{C}^{p+1}(G, V)_{M} .
\end{aligned}
$$


Proof. The identities follow since $\lambda_{t}^{(p)} \circ \partial_{i}=\partial_{i} \circ \lambda_{t}^{(p+1)}: E_{p} G \rightarrow E_{p-1} G$ for $0 \leq i<p$ (but usually not for $i=p$ ), and $\nu_{p-1} \circ \partial_{i}=\partial_{i} \circ \nu_{p}: B_{p} G \rightarrow E_{p-1} G$ for $0 \leq i<p$ (but usually not for $i=p)$.

Proof of Theorem 4.8, Let $\alpha \in \mathrm{C}^{p}(A, V)=\Gamma\left(V \otimes \wedge^{p} A^{*}\right)$. Then

$$
R_{G}(\alpha)=(-1)^{p} \mathrm{q} \circ(\delta \circ \mathrm{k})^{p} \mathrm{i}(\alpha) .
$$

In this expression, the leftmost $\delta$ is the map $\delta=\sum_{i=0}^{p}(-1)^{i} \partial_{i}^{*}: \mathrm{D}^{p-1,0}(G, V)_{M} \rightarrow \mathrm{D}^{p, 0}(G, V)_{M}$. Using $\mathrm{q} \circ \mathrm{k}=0$ and Lemma 4.11, we have that $\mathrm{q} \circ \partial_{i}^{*} \circ \mathrm{k}=\partial_{i}^{*} \circ \mathrm{q} \circ \mathrm{k}=0$ for $i<p$. Hence the composition $\mathrm{q} \circ \delta$ may be replaced with $(-1)^{p} \mathrm{q} \circ \partial_{p}^{*}=(-1)^{p} \nu_{p}^{*} \circ \partial_{p}^{*}$, leading to

$$
R_{G}(\alpha)=\nu_{p}^{*} \circ \partial_{p}^{*} \circ \mathrm{k} \circ(\delta \circ \mathrm{k})^{p-1} \mathrm{i}(\alpha) .
$$

If $p>1$, consider the leftmost product $\mathrm{k} \circ \delta \circ \mathrm{k}: \mathrm{D}^{p-1,2}(G, V)_{M} \rightarrow \mathrm{D}^{p-1,0}(G, V)_{M}$. Using $\mathrm{k} \circ \mathrm{k}=0$ and Lemma 4.11 again, $\mathrm{k} \circ \partial_{i}^{*} \circ \mathrm{k}=\partial_{i}^{*} \circ \mathrm{k} \circ \mathrm{k}=0$ for $i<p-1$; hence we may replace this expression with $(-1)^{p-1} \mathrm{k} \circ \partial_{p-1}^{*} \circ \mathrm{k}$. Continuing in this way, we arrive at

$$
R_{G}(\alpha)=(-1)^{p(p-1) / 2}\left(\nu_{p}^{*} \circ \partial_{p}^{*} \circ \mathrm{k} \circ \partial_{p-1}^{*} \circ \mathrm{k} \circ \cdots \circ \partial_{1}^{*} \circ \mathrm{k}\right) \mathrm{i}(\alpha) .
$$

But k: $\mathrm{D}^{p, q}(G, V)_{M} \rightarrow \mathrm{D}^{p, q-1}(G, V)_{M}$ is given by $(-1)^{p}$ times pull-back under the map $\lambda^{(p)}:[0,1] \times E_{p} G \rightarrow E_{p} G$, followed by integration over $[0,1]$. The signs for k's cancel the $(-1)^{p(p-1) / 2}$, and the resulting expression reads as

$$
\begin{aligned}
R_{G}(\alpha) & =\int_{[0,1]^{p}}\left(\nu_{p}^{*} \circ \partial_{p}^{*} \circ\left(\lambda^{(p)}\right)^{*} \circ \partial_{p-1}^{*} \circ\left(\lambda^{(p-1)}\right)^{*} \circ \cdots \circ \partial_{1}^{*} \circ\left(\lambda^{(1)}\right)^{*}\right) \mathrm{i}(\alpha) \\
& =\int_{[0,1]^{p}}\left(\gamma^{(p)}\right)^{*} \alpha^{L},
\end{aligned}
$$

where we used Lemma 4.10 and $\mathrm{i}(\alpha)=\alpha^{L}$.

4.5. Example: the pair groupoid. Let Pair $(M) \rightrightarrows M$ be the pair groupoid of the manifold $M$, with associated Lie algebroid the tangent bundle $T M$. Here $B_{p} G \cong M^{p+1}$, and $\mathrm{C}^{\bullet}(\operatorname{Pair}(M))_{M}=C^{\infty}\left(M^{\bullet+1}\right)_{M}$ is the Alexander-Spanier complex, with differential

$$
(\delta f)\left(m_{0}, \ldots, m_{p+1}\right)=\sum_{i=0}^{p+1}(-1)^{i} f\left(m_{0}, \ldots, m_{i-1}, m_{i+1}, \ldots, m_{p+1}\right),
$$

while $C^{\bullet}(T M)=\Omega^{\bullet}(M)$ is the usual de Rham complex. The Van Est differentiation is given on functions of the form $f=f_{0} \otimes \cdots \otimes f_{p}$ with $f_{i} \in C^{\infty}(M)$ by

$$
\mathrm{VE}\left(f_{0} \otimes \cdots \otimes f_{p}\right)=f_{0} \mathrm{~d} f_{1} \wedge \cdots \wedge \mathrm{d} f_{p}
$$

For the integration, choose an affine connection on $M$. For $m_{0}, m_{1} \in M$ sufficiently close, let $\rho\left(m_{0}, m_{1}\right):[0,1] \rightarrow M$ be the geodesic starting at $m_{0}$ and ending at $m_{1}$. Generalize to maps $\rho^{(p)}\left(m_{0}, \ldots, m_{p}\right):[0,1]^{p} \rightarrow M$, given by $\rho$ for $p=1$ and inductively by

$$
\rho_{t_{1}, \ldots, t_{p}}^{(p)}\left(m_{0}, \ldots, m_{p}\right)=\rho_{t_{1}}\left(m_{0}, \rho_{t_{2}, \ldots, t_{p}}^{(p-1)}\left(m_{1}, \ldots m_{p}\right)\right)
$$

The van Est integration $R_{G}$ takes $\alpha \in \Omega^{p}(M)$ to the (germ of a) function $\left(m_{0}, \ldots, m_{p}\right) \mapsto$ $\int_{[0,1]^{p}}\left(\rho^{(p)}\left(m_{0}, \ldots, m_{p}\right)\right)^{*} \alpha$. 


\section{VAN EST MAPS FOR LIE GROUPOID ACTIONS ON MANIFOLDS}

In his paper [7], Crainic proved a general van Est theorem for (proper) groupoid actions on manifolds $Q$. We explain how to generalize the differentiation and integration maps for cochains to this context. The construction of a horizontal homotopy operator for the double complex will require the additional data of a Haar distribution.

5.1. Haar distributions. By a (left-invariant) Haar distribution on a Lie groupoid $G \rightrightarrows M$, we mean a family $\mu=\left\{\mu_{m}\right\}$ of distributions on the t-fibers

$$
\mu_{m} \in \mathcal{D}^{\prime}\left(\mathrm{t}^{-1}(m)\right), \quad m \in M,
$$

such that:

- The family depends smoothly on $m$, in the sense that for any compactly supported function $f \in C^{\infty}(G)$ the integral over t-fibers defines a smooth function

$$
m \mapsto \int_{a \in \mathrm{t}^{-1}(m)} f(a) \mu_{m}(a) .
$$

- The family is left-invariant, in the sense that $\left(l_{g}\right)_{*} \mu_{m}=\mu_{g \cdot m}$ for all $g \in G$ with $\mathrm{s}(g)=m$. The Haar distribution is called properly supported if $\mathrm{t}$ restricts to a proper map $\operatorname{supp}(\mu) \rightarrow M$; in particular, this means that the individual distributions $\mu_{m}$ are compactly supported. It is called normalized if furthermore $\int_{\mathrm{t}^{-1}(m)} \mu_{m}=1$ for all $m$, and non-negative if the integral (39) is non-negative for all $f \geq 0$. A Haar distribution is called a Haar density if it is smooth; by left-invariance, these are equivalent to smooth sections of the density bundle of $A=\operatorname{Lie}(G)$. It is known [7, 9, 26] that if the Lie groupoid is proper, in the sense that (t, s):G $\rightarrow M \times M$ is a proper map, then $G$ admits a properly supported, non-negative, normalized Haar density.

As shown by Crainic [7, Proof of Proposition 1], a properly supported normalized Haar distribution $\mu$ for a proper groupoid $G \rightrightarrows M$ defines a homotopy operator for the groupoid cochain complex $\mathrm{C}(G, V)$, for any $G$-representation $V$ :

$$
(\mathrm{h} \sigma)\left(g_{1}, \ldots, g_{p-1}\right)=(-1)^{p} \int_{a \in \mathrm{t}^{-1}(m)} a \cdot \sigma\left(g_{1}, \ldots, g_{p-1}, a\right) \mu_{m}(a)
$$

where $m=\mathrm{s}\left(g_{p-1}\right)$. (Actually, [7] only considers Haar densities, but the calculation for distributions is exactly the same.) Thus $[\mathrm{h}, \delta]=1-\mathrm{i} \circ \mathrm{p}$, where $\mathrm{i}$ is the inclusion of invariant sections $\Gamma(V)^{G}$, while $\mathrm{p}$ takes a section $\tau \in \Gamma(V)$ to the invariant section obtained by averaging:

$$
(\mathrm{p} \tau)_{m}=\int_{a \in \mathrm{t}^{-1}(m)} a \cdot \tau(\mathrm{s}(a)) \mu_{m}(a) .
$$

Examples 5.1. (a) For a Lie group $G$, every Haar distribution is automatically smooth, and is obtained by left translation of an element of the density space of $T_{e} G=\mathfrak{g}$. The groupoid $G \rightrightarrows$ pt is proper (and its Haar measure is properly supported) if and only if $G$ is compact.

(b) The pair groupoid $\operatorname{Pair}(M)=M \times M \rightrightarrows M$ is proper. Its t-fibers are $\mathrm{t}^{-1}(m)=$ $\{m\} \times M \cong M$; under this identification, the left-action of elements $a=\left(m^{\prime}, m\right)$ on Pair $(M)$ corresponds to the trivial diffeomorphism of $M$. Hence, any fixed $\nu \in \mathcal{D}^{\prime}(M)$ defines a Haar distribution with $\mu_{m}=\nu$ independent of $m$; it is proper if $\nu$ has compact 
support, and normalized if $\int \nu=1$. In particular, we may take $\nu$ to be the deltadistribution at any given base point $z \in M$. The resulting homotopy for the complex $C^{\bullet}(\operatorname{Pair}(M))=C^{\infty}\left(M^{\bullet+1}\right)$ is the standard one:

$$
(\mathrm{h} f)\left(m_{0}, \ldots, m_{p-1}\right)=(-1)^{p} f\left(m_{0}, \ldots, m_{p-1}, z\right) .
$$

(c) Let $\mathcal{U}=\left\{U_{i}\right\}$ be an open cover of a manifold $M$, and put $X=\sqcup_{i} U_{i}$. The associated Cech groupoid $X \times_{M} X \rightrightarrows X$, with the groupoid structure induced from the pair groupoid Pair $(X)$, is proper. A locally finite partition of unity $\left\{\chi_{i}\right\}$ subordinate to the cover $\mathcal{U}$ defines a normalized properly supported Haar distribution: the t-fiber of $m \in U_{i}$ is a disjoint union of copies of $\{m\}$ (one for each $U_{j}$ containing $m$ ), and the Haar density on this discrete set is given by the sequence $\left\{\chi_{j}(m)\right\}$. The invariant elements of $\mathrm{C}^{0}\left(X \times_{M} X\right)=C^{\infty}(X)$ are pullback of functions on $M$, and the homotopy operator on the Cech complex $\mathrm{C}\left(X \times_{M} X\right)$ is the standard one [3, Chapter 2.8], cf. Example 2.2.

Suppose that $G \rightrightarrows M$ is a Lie groupoid, and that $Q$ is a $G$-manifold, with anchor map $\Phi: Q \rightarrow M$. The action is called proper if the action groupoid $G \ltimes Q \rightrightarrows Q$ is proper. The t-fiber of $x \in Q$ in the action groupoid is canonically identified with the t-fiber of $\Phi(x) \in M$ in the groupoid $G$; hence a Haar distribution $\mu$ for the action groupoid amounts to a family of distributions

$$
\mu_{x} \in \mathcal{D}^{\prime}\left(\mathrm{t}^{-1}(\Phi(x))\right), \quad x \in Q
$$

with the following invariance property:

$$
\mu_{a . x}=\left(l_{a}\right)_{*} \mu_{x}, \quad x \in Q, a \in G .
$$

If the action is proper, there exists a properly supported normalized Haar distribution.

Examples 5.2. (a) For the action of $G$ on $Q=G$ by left translation, we may take (42) to be the collection of delta-distributions

$$
\mu_{g}=\delta_{g} \in \mathcal{D}^{\prime}\left(\mathrm{t}^{-1}(\mathrm{t}(g)) .\right.
$$

(b) Let $G$ be a Lie group, and consider the homogeneous space $Q=G / K$ where $K$ a compact subgroup. Let $\delta_{K} \in \mathcal{D}^{\prime}(G)$ be the push-forward of the normalized Haar density on $K$. Then the family of distributions $\mu_{g K}=\left(l_{g}\right)_{*} \delta_{K}$ defines a normalized, properly supported Haar distribution.

5.2. The van Est double complex. For the rest of this section, suppose that $G \rightrightarrows M$ is a Lie groupoid acting on a manifold $Q$, with moment map $\Phi: Q \rightarrow M$ a submersion. The fibers of $\Phi$ define a $G$-invariant foliation $\mathcal{F}$ of $Q$, and a corresponding $G$-equivariant Lie algebroid $T_{\mathcal{F}} Q=\operatorname{ker}(T \Phi) \subseteq T Q$. For any vector bundle $V \rightarrow M$, we obtain a 'fiberwise trivial' Lie algebroid representation of $T_{\mathcal{F}} Q$ on the vector bundle $\Phi^{*} V=Q \times_{M} V \rightarrow Q$; given a $G$ representation on $V \rightarrow M$, this representation is compatible with the $G$-action on $\Phi^{*} V$. It defines a foliated de Rham complex

$$
\Omega_{\mathcal{F}}^{\bullet}\left(Q, \Phi^{*} V\right)=\Gamma\left(\Phi^{*} V \otimes \wedge^{\bullet} T_{\mathcal{F}}^{*} Q\right) .
$$

The invariant subcomplex $\Omega_{\mathcal{F}}^{\bullet}\left(Q, \Phi^{*} V\right)^{G}$ consists of sections $\phi \in \Gamma\left(\Phi^{*} V \otimes \wedge T_{\mathcal{F}}^{*} Q\right)$ with the equivariance property $\phi(g \cdot q)=g \cdot \phi(q)$. (If $Q=G$ with the $G$-action by left translation, this 
is the complex of left-invariant $\mathrm{t}^{*} V$-valued forms on $G$, and is identified with $\mathrm{C}(A, V)$. If $G$ is a Lie group and $Q=G / K$, this is the de Rham complex $\Omega(G / K, V)^{G} \cong \mathrm{C}(\mathfrak{g}, V)_{K \text {-basic }}$. Let

$$
\Phi_{p}: B_{p} G \times_{M} Q \rightarrow B_{p} G
$$

be the natural projection (given by $\Phi$ if $p=0$ ). The foliation of $Q$ extends to foliations $\mathcal{F}$ of $B_{p} G \times{ }_{M} Q$ given by the fibers of $\Phi_{p}$.

We obtain a simplicial Lie algebroid $T_{\mathcal{F}}\left(B_{p} G \times_{M} Q\right)=B_{p} G \times_{M} T_{\mathcal{F}} Q$, together with a (fiberwise trivial) representation on the vector bundle $\Phi_{p}^{*}\left(B_{p} G \times{ }_{M} V\right) \rightarrow B_{p} G \times{ }_{M} Q$. Following Crainic [7, define a double complex

$$
\mathrm{D}^{p, q}(Q, V)=\mathrm{C}^{q}\left(T_{\mathcal{F}}\left(B_{p} G \times_{M} Q\right), \Phi_{p}^{*}\left(B_{p} G \times_{M} V\right)\right.
$$

with the usual simplicial differential $\delta$, and with $\mathrm{d}=(-1)^{p} \mathrm{~d}_{C E}$. Its elements may be regarded as maps $\beta: B_{p} G \rightarrow \Omega_{\mathcal{F}}^{q}\left(Q, \Phi^{*} V\right)$, with

$$
\beta\left(g_{1}, \ldots, g_{p}\right) \in \Omega^{q}\left(\Phi^{-1}(m), V_{m}\right), \quad m=\mathrm{s}\left(g_{p}\right) .
$$

with $\mathrm{d}=(-1)^{p} \mathrm{~d}_{R h}$ and $\delta$ as in (19). This double complex comes with a horizontal augmentation map i: $\Omega_{\mathcal{F}}\left(Q, \Phi^{*} V\right)^{G} \rightarrow \mathrm{D}^{0, \bullet}(Q, V)$ given by the inclusion of invariant elements, and a vertical augmentation map j: $\mathrm{C}(G, V) \rightarrow \mathrm{D}^{\bullet}, 0(Q, V)=\Gamma\left(\Phi_{p}^{*}\left(B_{p} G \times_{M} V\right)\right)$ given by pullback under the bundle projection $B_{p} G \times_{M} Q \rightarrow B_{p} G$. For the total complex this gives cochain maps

$$
\mathrm{C}^{\bullet}(G, V) \stackrel{\mathrm{j}}{\longrightarrow} \operatorname{Tot}^{\bullet}(\mathrm{D}(Q, V)) \stackrel{\mathrm{i}}{\longleftarrow} \Omega_{\mathcal{F}}^{\bullet}\left(Q, \Phi^{*} V\right)^{G} .
$$

5.3. Differentiation. Assuming that the $G$-action on $Q$ is proper, we may choose a properly supported normalized Haar distribution $\mu=\left\{\mu_{x}\right\}$ for $G \ltimes Q \rightrightarrows Q$. It determines a horizontal homotopy $\mathrm{h}$ on the double complex $\mathrm{D}^{\bullet \bullet \bullet}(Q, V)$; thus $[\delta, \mathrm{h}]=1-\mathrm{i} \circ \mathrm{p}$, where $\mathrm{p}$ is the averaging map with respect to $\mu$. These are given by (40) and (41), replacing $G$ with $G \ltimes Q$ and $V$ with $\Phi^{*} V$. Explicitly:

$$
(\mathrm{h} \beta)\left(g_{1}, \ldots, g_{p-1}\right)_{x}=(-1)^{p} \int_{\mathrm{t}(a)=m} a \cdot \beta\left(g_{1}, \ldots, g_{p-1}, a\right)_{a^{-1} \cdot x} \mu_{x}(a)
$$

for $m=\mathrm{s}\left(g_{p-1}\right)$ and all $x \in \Phi^{-1}(m)$, and

$$
(\mathrm{p} \phi)_{x}=\int_{\mathrm{t}(a)=\Phi(x)} a \cdot \phi_{a^{-1} \cdot x} \mu_{x}(a) .
$$

for $\phi \in \mathrm{D}^{0, q}(Q, V)=\mathrm{C}^{q}\left(T_{\mathcal{F}} Q, \Phi^{*} V\right)$. The Perturbation Lemma 2.1 defines a homotopy inverse $\mathrm{p}^{\prime}=\mathrm{p} \circ(1+\mathrm{dh})^{-1}$ to $\mathrm{i}$, and a cochain map

$$
\mathrm{VE}_{Q}=\mathrm{p} \circ(1+\mathrm{dh})^{-1} \circ \mathrm{j}: \mathrm{C}^{\bullet}(G, V) \rightarrow \Omega_{\mathcal{F}}^{\bullet}\left(Q, \Phi^{*} V\right)^{G} .
$$

Example 5.3. If $Q=G$ with the left-action of $G$, and using the Haar distribution from Example 5.2 (a) , we recover the homotopy operator $\mathrm{h}$ and projection $\mathrm{p}$ from Section 4 . As we saw, this leads to the van Est differentiation map $\mathrm{VE}_{G}$ of Weinstein-Xu.

Example 5.4. Let $G$ be a Lie group, $K$ a compact Lie subgroup, and $Q=G / K$. Let $\mu$ be the Haar distribution from Example 5.2 (b) , thus $\mu_{g K}(a)=\delta_{K}\left(g^{-1} a\right)$. Making a change of variables $a=g k$, we recover the homotopy operator $\mathrm{h}$ and projection $\mathrm{p}$ from Section 3 , leading to the differentiation map $\mathrm{VE}_{G / K}$ discussed there. 
5.4. Integration. Suppose that the submersion $\Phi: Q \rightarrow M$ admits a section $r: M \rightarrow Q$, i.e, $\Phi \circ r=\operatorname{id}_{M}$. Fixing $r$ we can think of $M$ as a submanifold as a submanifold of $Q$. Choose a tubular neighborhood embedding $\nu(Q, M) \rightarrow M$, taking the fibers of the normal bundle to the $\Phi$-fibers, to define a germ (along $[0,1] \times M$ ) of a retraction $\lambda:[0,1] \times Q \rightarrow Q$, with $\lambda_{t_{1} t_{2}}=\lambda_{t_{1}} \circ \lambda_{t_{2}}$, where

$$
\lambda_{0}=r \circ \Phi, \quad \lambda_{1}=\operatorname{id}_{Q}, \Phi \circ \lambda_{t}=\Phi, \quad \lambda_{t} \circ r=r .
$$

In turn, it gives a homotopy operator $T$ on the localized foliated de Rham complex $\Omega_{\mathcal{F}}\left(Q, \Phi^{*} V\right)_{M}$.

The discussion from Section 4.4 (for the case $Q=G$ ) extends to this setting in a straightforward fashion: One obtains a homotopy operator $\mathrm{k}=(-1)^{p} T$ on the double complex $\mathrm{D}(Q, V)_{M}$, with $\mathrm{k} \circ \mathrm{k}=0$ and $\mathrm{q} \circ \mathrm{k}=0$; in turn, this defines a homotopy inverse $\mathrm{q}^{\prime}=$ $\mathrm{q} \circ(1+\delta \mathbf{k})^{-1}: \operatorname{Tot}(\mathrm{D}(Q, V)) \rightarrow \mathrm{C}(G, V)$ to $\mathbf{j}$, and the resulting van Est integration map

$$
R_{Q}=\mathrm{q} \circ(1+\delta \mathrm{k})^{-1} \circ \mathrm{i}: \Omega_{\mathcal{F}}\left(Q, \Phi^{*} V\right)^{G} \rightarrow \mathrm{C}(G, V) .
$$

is described by the formula

$$
R_{Q}(\alpha)\left(g_{1} \ldots, g_{p}\right)=\int_{[0,1]^{p}} \gamma^{(p)}\left(g_{1}, \ldots, g_{p}\right)^{*} \alpha,
$$

for $\alpha \in \Omega_{\mathcal{F}}^{p}\left(Q, \Phi^{*} V\right)^{G}$. Here $\gamma^{(p)}\left(g_{1}, \ldots, g_{p}\right):[0,1]^{p} \rightarrow Q$ is defined similar to (37):

$$
\gamma_{t_{1}, \ldots, t_{p}}^{(p)}\left(g_{1}, \ldots, g_{p}\right)=\lambda_{t_{1}}\left(g_{1} \lambda_{t_{2}}\left(g_{2} \cdots \lambda_{t_{p}}\left(g_{p} \cdot r\left(\mathrm{~s}\left(g_{p}\right)\right)\right) \cdots\right)\right) .
$$

If the $G$-action on $Q$ is furthermore proper, we also have the differentiation map $\mathrm{VE}_{Q}$, defined by the properly supported normalized Haar distribution $\mu$. In general, the van Est integration map $R_{Q}$ defined by $\lambda_{t}$ need not be a right inverse to the differentiation map $\mathrm{VE}_{Q}$ - the compatibility conditions of Lemma 2.3 need not be satisfied, in general. One general setting where they are satisfied is the following.

Proposition 5.5. Suppose $\mu=\left\{\mu_{x}\right\}$ is a properly supported normalized Haar distribution for $G \ltimes Q \rightrightarrows Q$ with the property

$$
\operatorname{supp}(\mu) \subseteq G \times{ }_{M} r(M)
$$

(as a subset of $G \ltimes Q=G \times{ }_{M} Q$ ). Then the conditions of Lemma 2.3 are satisfied: that is, $\mathrm{h} \circ \mathrm{k}=0, \mathrm{p} \circ \mathrm{k}=0$.

Proof. The condition $\operatorname{supp}(\mu) \subseteq G \times_{M} r(M)$ is equivalent to the requirement that for all $x \in Q$,

$$
\operatorname{supp}\left(\mu_{x}\right) \subseteq\left\{a \in G \mid a^{-1} \cdot x \in r(M)\right\} .
$$

$\left.\operatorname{But}(\mathrm{k} \beta)\left(g_{1}, \ldots, g_{p}\right)\right|_{r(M)}=0$, by the usual properties of the de Rham homotopy operator. On the other hand, the explicit formula (46) for the homotopy operator $\mathrm{h}$ shows that for all $a \in \operatorname{supp}\left(\mu_{x}\right)$,

$$
(\mathrm{k} \beta)\left(g_{1}, \ldots, g_{p}\right)_{a^{-1} \cdot x}=\left.0 \Rightarrow(\mathrm{hk} \beta)\left(g_{1}, \ldots, g_{p-1}\right)\right|_{x}=0 .
$$

Hence $\mathrm{h} \circ \mathrm{k}=0$ if (49) holds true, and likewise $\mathrm{p} \circ \mathrm{k}=0$.

This result 'explains' Propositions 3.4 and 4.7 , 
Example 5.6. Let $G$ be a Lie group, $K$ a compact subgroup, and $Q=G / K$. The Haar distribution $\mu_{g K}=\left(l_{g}\right)_{*} \delta_{K}$ is supported in $g K \subseteq G$, which is the set of all $a \in G$ such that $a^{-1} g K=e K$, hence (49) holds true. In fact,

$$
\operatorname{supp}(\mu)=G \times e K \subseteq G \ltimes G / K .
$$

Example 5.7. Let $G \rightrightarrows M$ be any Lie groupoid, and $Q=G$, with the Haar distribution $\mu_{g}=\delta_{g}$. Then (49) holds true, in fact,

$$
\operatorname{supp}(\mu)=G \times_{M} M \subseteq G \ltimes G .
$$

\section{Appendix A. Proof of Theorem 4.4}

Taking $Q=G$ in (25), we have $p+1$ commuting $G$-actions on $E_{p} G=B_{p} G \times{ }_{M} G$; these commute with the principal action and descend to the actions on $B_{p} G$. The projection $\pi_{p}: E_{p} G \rightarrow M$ intertwines each of these actions with the trivial action on $M$; hence we obtain commuting $G$-actions on the vector bundles

$$
\pi_{p}^{*}\left(V \otimes \wedge^{q} A^{*}\right)=E_{p} G \times_{M}\left(V \otimes \wedge^{q} A^{*}\right) \subseteq E_{p} G \times\left(V \otimes \wedge^{q} A^{*}\right),
$$

using the trivial action on the $V \otimes \wedge^{q} A^{*}$ factor. The infinitesimal action gives covariant derivatives $\nabla_{\xi}^{(i)}$ on $\mathrm{D}^{p, q}(G, V)=\Gamma\left(\pi_{p}^{*}\left(\wedge^{q} A^{*} \otimes V\right)\right)$; the derivatives for different $i$ 's commute. They 'lift' the operators $\nabla_{\xi}^{(i)}$ on $C^{p}(G, V)=\Gamma\left(B_{p} G \times_{M} V\right)$ introduced earlier.

Lemma A.1. (a) The maps j: $\mathrm{C}^{p}(G, V) \rightarrow \mathrm{D}^{p, 0}(G, V)$ intertwine $\nabla_{\xi}^{(i)}$ for $i=0, \ldots, p$.

(b) The operators $\nabla_{\xi}^{(i)}$ on the double complex commute with the vertical differential $\mathrm{d}$, and also with contractions $\iota_{\zeta}, \zeta \in \Gamma(A)$ and Lie derivatives $\mathcal{L}_{\zeta}$.

(c) The maps $\mathrm{h}=(-1)^{p} h_{p-1}^{*}: \mathrm{D}^{p, q}(G, V) \rightarrow \mathrm{D}^{p-1, q}(G, V)$ intertwine $\nabla_{\xi}^{(i)}$ for $i=0, \ldots, p-$ 1, while

$$
\mathcal{L}_{\xi} \circ \mathrm{h}=\mathrm{h} \circ\left(\nabla_{\xi}^{(p)}+\mathcal{L}_{\xi}\right)
$$

Proof. (a) follows from the equivariance of the map $\kappa_{p}$ with respect to the $i$-th action.

(b) Since $\iota_{\zeta}$ is equivariant for $i$-th action, it intertwines the operators $\nabla_{\xi}^{(i)}$. Next, since $\kappa_{p}: E_{p} G \rightarrow B_{p} G$ is equivariant for the $i$-th action; the foliation $\mathcal{F}$ of $E_{p} G$ is preserved; i.e., the infinitesimal action of $\Gamma(A)$ is by infinitesimal automorphisms of the Lie algebroid $T_{\mathcal{F}} E_{p} G$. It follows that the action on $\mathrm{D}^{p, \bullet}(G, V)$ preserves the differential $\mathrm{d}_{C E}$ and hence also $\mathrm{d}=(-1)^{p} \mathrm{~d}_{C E}$. Finally, since $\mathcal{L}_{\zeta}=\left[\mathrm{d}_{C E}, \iota_{\zeta}\right]$ it also intertwines the Lie derivatives (for the principal $G$-action); alternatively this follows directly because the $i$-th action commutes with the principal action.

(c) The first part follows since the maps

$$
h_{p-1}: E_{p-1} G \rightarrow E_{p} G,\left(g_{1}, \ldots, g_{p-1} ; g\right) \mapsto\left(g_{1}, \ldots, g_{p-1}, g ; \mathrm{s}(g)\right)
$$

(see (11)) are equivariant for the actions labeled by $i=0, \ldots, p-1$. For (51), we need to consider both the generating vector fields $\xi^{(p)}$ for the $p$-th $G$-action and the generators $\xi_{E_{p} G} \in \mathfrak{X}\left(E_{p} G\right)$ of the principal action. In terms of $E_{p} G=B_{p} G \times{ }_{M} G$,

$$
\xi^{(p)}=\left(\xi^{L, p},-\xi^{R}\right), \quad \xi_{E_{p} G}=\left(0, \xi^{L}\right),
$$


where $\xi^{L, p}$ is the left-invariant vector field sitting on the last $G$-factor of $B_{p} G$. Since $\xi^{L} \sim_{u \circ s} \xi^{L}-\xi^{R}$ (where $u: M \rightarrow G$ is the inclusion of units), we see that

$$
\xi_{E_{p-1} G} \sim_{h_{p-1}} \xi_{E_{p} G}+\xi^{(p)},
$$

which implies Equation (51).

We are now in position to give the proof of Theorem 4.4,

Proof of Theorem 4.4. On elements of $C^{p}(G, V)=\Gamma\left(B_{p} G \times{ }_{M} V\right)$, we have that

$$
\mathrm{VE}_{G}=(-1)^{p} \mathrm{p} \circ(\mathrm{dh})^{p} \circ \mathrm{j} .
$$

Using

$$
\begin{gathered}
\mathrm{j}=\kappa_{p}^{*}: \mathrm{C}^{p}(G, V) \rightarrow \mathrm{D}^{p, 0}(G, V), \\
\mathrm{dh}=-\mathrm{d}_{C E} \circ h_{i-1}^{*}: \mathrm{D}^{i, p-i}(G, V) \rightarrow \mathrm{D}^{i-1, p-i+1}(G, V), \\
\mathrm{p}=u^{*}: \mathrm{D}^{0, p}(G, V) \rightarrow \mathrm{C}^{p}(A, V),
\end{gathered}
$$

this means that $\mathrm{VE}_{G}=u^{*} \circ \mathrm{d}_{C E} \circ h_{0}^{*} \circ \mathrm{d}_{C E} \circ \cdots \circ h_{p-1}^{*} \circ \kappa_{p}^{*}$. Given $\xi_{1}, \ldots, \xi_{p} \in \Gamma(A)$ and $\sigma \in \Gamma\left(B_{p} G \times_{M} V\right)$, we want to compute

$$
\operatorname{VE}_{G}(\sigma)\left(\xi_{1}, \ldots, \xi_{p}\right)=\iota_{\xi_{p}} \cdots \iota_{\xi_{1}} u^{*} \mathrm{~d}_{C E} h_{0}^{*} \mathrm{~d}_{C E} h_{1}^{*} \cdots \mathrm{d}_{C E} h_{p-1}^{*} \kappa_{p}^{*} \sigma .
$$

Our strategy is to move the variables $\xi_{p}, \ldots, \xi_{1}$ to the right, while retaining their ordering (keeping $\xi_{i}$ to the left of $\xi_{j}$ if $i>j$ ). The commutators of contractions $\iota_{\xi}$ with $\mathrm{d}_{C E}$ produces Lie derivatives $\mathcal{L}_{\xi}=\left[\iota_{\xi}, \mathrm{d}_{C E}\right]$. Using Lemma A.1 and $\mathcal{L}_{\xi} \circ \kappa_{p}^{*}=0$, we find

$$
\mathcal{L}_{\xi} \circ h_{i-1}^{*} \cdots \mathrm{d}_{C E} \circ h_{p-1}^{*} \circ \kappa_{p}^{*}=h_{i-1}^{*} \circ \cdots \mathrm{d}_{C E} \circ h_{p-1}^{*} \circ \kappa_{p}^{*} \circ \widehat{\nabla}_{\xi}^{(i)}
$$

where we introduced the hat notation

$$
\widehat{\nabla}_{\xi}^{(i)}=\nabla_{\xi}^{(i)}+\ldots+\nabla_{\xi}^{(1)}
$$

corresponding to the diagonal action for the actions labeled $1, \ldots, i$. (Note that the 0 -th action is not included.) We therefore obtain

$$
\begin{aligned}
\mathrm{VE}_{G}(\sigma)\left(\xi_{1}, \ldots, \xi_{p}\right) & =u^{*} h_{0}^{*} \cdots h_{p-1}^{*} \kappa_{p}^{*} \sum_{s \in \mathfrak{S}_{p}} \operatorname{sign}(s) \widehat{\nabla}_{\xi_{p}}^{(s(p))} \cdots \widehat{\nabla}_{\xi_{1}}^{(s(1))} \sigma \\
& =\left.\left(\sum_{s \in \mathfrak{S}_{p}} \operatorname{sign}(s) \widehat{\nabla}_{\xi_{p}}^{(s(p))} \cdots \widehat{\nabla}_{\xi_{1}}^{(s(1))} \sigma\right)\right|_{M} ;
\end{aligned}
$$

here the second equality follows since the composition $\kappa_{p} \circ h_{p-1} \circ \cdots \circ h_{0} \circ u$ is just the inclusion $M \rightarrow B_{p} G$. To complete the proof, we argue that

$$
\sum_{s \in \mathfrak{S}_{p}} \operatorname{sign}(s) \widehat{\nabla}_{\xi_{p}}^{(s(p))} \cdots \widehat{\nabla}_{\xi_{1}}^{(s(1))}
$$

is equal to a similar sum with all hats removed. Given $s \in \mathfrak{S}_{p}$, let $i=s^{-1}(p)$. Since

$$
\widehat{\nabla}_{\xi_{i}}^{(p)}=\nabla_{\xi_{i}}^{(p)}+\widehat{\nabla}_{\xi_{i}}^{(p-1)}
$$


we see that the product

$$
\widehat{\nabla}_{\xi_{p}}^{(s(p))} \cdots\left(\widehat{\nabla}_{\xi_{i}}^{(p)}-\nabla_{\xi_{i}}^{(p)}\right) \cdots \widehat{\nabla}_{\xi_{1}}^{(s(1))}
$$

coincides with the corresponding expression for the permutation $s^{\prime}$, given as the composition of $s$ with the transposition of the indices $p, p-1$. Since $s, s^{\prime}$ have opposite signs, it follows that (52) does not change when we remove the hats from all $\widehat{\nabla}_{\xi_{i}}^{(s(i))}$ for which $s(i)=p$.

Having done so, and assuming $p>2$, consider for a given $s \in \mathfrak{S}_{p}$ the indices $i, j$ for which $s(i)=p, s(j)=p-1$. (If $p=2$, we may simply put $\widehat{\nabla}_{\xi}^{(1)}=\nabla_{\xi}^{(1)}$, completing the proof.) An argument similar to the first step shows that the expression

$$
\widehat{\nabla}_{\xi_{p}}^{(s(p))} \cdots \nabla_{\xi_{i}}^{(p)} \cdots\left(\widehat{\nabla}_{\xi_{j}}^{(p-1)}-\nabla_{\xi_{i}}^{(p-1)}\right) \cdots \widehat{\nabla}_{\xi_{1}}^{(s(1))}
$$

coincides with a similar expression for the composition of $s$ with transposition of the indices $p-1, p-2$. (We wrote (53) for the case that $i>j$; of course, if $i<j$ the $\nabla_{\xi_{i}}^{(p)}$ would appear to the right of $\widehat{\nabla}_{\xi_{j}}^{(p-1)}-\nabla_{\xi_{j}}^{(p-1)}$.) Since those permutations have opposite signs, it shows that we may also remove the hat from the factors $\nabla_{\xi_{j}}^{(s(j)}$ with $s(j)=p-1$. Removing all the hats in this manner, we have proved the Weinstein-Xu formula

$$
\mathrm{VE}_{G}(\sigma)\left(\xi_{1}, \ldots, \xi_{p}\right)=\left.\left(\sum_{s \in \mathfrak{S}_{p}} \operatorname{sign}(s) \nabla_{\xi_{p}}^{(s(p))} \cdots \nabla_{\xi_{1}}^{(s(1))} \sigma\right)\right|_{M}
$$

\section{REFERENCES}

1. C. Abad and M. Crainic, The Weil algebra and the Van Est isomorphism, Ann. Inst. Fourier (Grenoble) 61 (2011), no. 3, 927-970.

2. A. Borel, Semisimple groups and Riemannian symmetric spaces, Texts and Readings in Mathematics, vol. 16, Hindustan Book Agency, New Delhi, 1998. MR 1661166

3. R. Bott and L. Tu, Differential forms in algebraic topology, Graduate Texts in Mathematics, vol. 82, SpringerVerlag, New York, 1982.

4. R. Brown, The twisted Eilenberg-Zilber theorem, Simposio di Topologia (Messina, 1964), Edizioni Oderisi, Gubbio, 1965, pp. 33-37.

5. A. Cabrera and T. Drummond, Van Est isomorphism for homogeneous cochains, Pacific J. Math. 287 (2017), no. 2, 297-336.

6. A. Cabrera, I. Marcut, and A. Salazar, On local integration of Lie brackets, Journal für die reine und angewandte Mathematik, to appear.

7. M. Crainic, Differentiable and algebroid cohomology, van Est isomorphisms, and characteristic classes, Comment. Math. Helv. 78 (2003), no. 4, 681-721.

8. M. Crainic and R.L. Fernandes, Lectures on integrability of Lie brackets, Lectures on Poisson geometry, Geom. Topol. Monogr., vol. 17, Geom. Topol. Publ., Coventry, 2011, pp. 1-107.

9. M. Crainic and J. N. Mestre, Orbispaces as differentiable stratified spaces, Lett. Math. Phys. 108 (2018), no. 3, 805-859. MR 3765980

10. J.-P. Dufour and N.T. Zung, Poisson structures and their normal forms, Progress in Mathematics, vol. 242, Birkhäuser Verlag, Basel, 2005.

11. J. L. Dupont, Simplicial de Rham cohomology and characteristic classes of flat bundles, Topology 15 (1976), no. 3, 233-245.

12. J. L. Dupont and A. Guichardet, à propos de l'article: "Sur la cohomologie réelle des groupes de Lie simples réels" [Ann. Sci. Ećole Norm. Sup. (4) 11 (1978), no. 2, 277-292] par Guichardet et D. Wigner, Ann. Sci. École Norm. Sup. (4) 11 (1978), no. 2, 293-295. MR 510553

13. V. Gugenheim, On the chain-complex of a fibration, Illinois J. Math. 16 (1972), 398-414. 
14. A. Guichardet, Cohomologie des groupes topologiques et des algèbres de Lie, Textes Mathématiques [Mathematical Texts], vol. 2, CEDIC, Paris, 1980.

15. A. Guichardet and D. Wigner, Sur la cohomologie réelle des groupes de Lie simples réels, Ann. Sci. École Norm. Sup. (4) 11 (1978), no. 2, 277-292. MR 510552

16. J.-C. Houard, An integral formula for cocycles of Lie groups, Ann. Inst. H. Poincaré Sect. A (N.S.) 32 (1980), no. 3, 221-247. MR 579961

17. Sze-tsen Hu, Cohomology theory in topological groups, Michigan Math. J. 1 (1952), 11-59. MR 0051244

18. D. Li-Bland and E. Meinrenken, On the van Est homomorphism for Lie groupoids, Enseign. Math. 61 (2015), no. 1-2, 93-137.

19. R. Mehta, Q-groupoids and their cohomology, Pacific J. Math. 242 (2009), no. 2, 311-332.

20. I. Moerdijk and J. Mrčun, Introduction to foliations and Lie groupoids, Cambridge Studies in Advanced Mathematics, vol. 91, Cambridge University Press, Cambridge, 2003.

21. M. Mostow and J. Perchik, Notes on Gelfand-Fuks cohomology and characteristic classes (lectures delivered by R. Bott), Proceedings of the eleventh annual holiday symposium, New Mexico State University, 1973, pp. $1-126$.

22. M. J. Pflaum, H. Posthuma, and X. Tang, The localized longitudinal index theorem for Lie groupoids and the van Est map, Adv. Math. 270 (2015), 223-262.

23. G. Segal, Classifying spaces and spectral sequences, Inst. Hautes Études Sci. Publ. Math. (1968), no. 34, $105-112$.

24. H. Shulman and D. Tischler, Leaf invariants for foliations and the Van Est isomorphism, J. Differential Geometry 11 (1976), no. 4, 535-546.

25. S. Świerczkowski, Cohomology of group germs and Lie algebras, Pacific J. Math. 39 (1971), 471-482. MR 0311746

26. Jean Louis Tu, La conjecture de Novikov pour les feuilletages hyperboliques, K-Theory 16 (1999), no. 2, $129-184$.

27. W. T. van Est, Group cohomology and Lie algebra cohomology in Lie groups. I, II, Nederl. Akad. Wetensch. Proc. Ser. A. 56 = Indagationes Math. 15 (1953), 484-492, 493-504.

28. _ On the algebraic cohomology concepts in Lie groups. I, II, Nederl. Akad. Wetensch. Proc. Ser. A. $\mathbf{5 8}$ = Indag. Math. 17 (1955), 225-233, 286-294.

29. Une application d'une méthode de Cartan-Leray, Nederl. Akad. Wetensch. Proc. Ser. A. 58 = Indag. Math. 17 (1955), 542-544.

30. A. Weinstein and P. Xu, Extensions of symplectic groupoids and quantization, J. Reine Angew. Math. 417 (1991), 159-189.

\author{
Department of Mathematics, University of Toronto (Canada) \\ E-mail address: mein@math.toronto.edu \\ Instituto de Matematica e Estatistica, Universidade Federal Fluminense (Brazil) \\ E-mail address: mariasalazar@id.uff.br
}

